\title{
A Finite Difference Method for the Variational $p$-Laplacian
}

\author{
Félix del Teso ${ }^{1}$ (D) Erik Lindgren ${ }^{2}$
}

Received: 12 April 2021 / Revised: 4 December 2021 / Accepted: 7 December 2021 /

Published online: 31 December 2021

(C) The Author(s) 2021

\section{Abstract}

We propose a new monotone finite difference discretization for the variational $p$-Laplace operator, $\Delta_{p} u=\operatorname{div}\left(|\nabla u|^{p-2} \nabla u\right)$, and present a convergent numerical scheme for related Dirichlet problems. The resulting nonlinear system is solved using two different methods: one based on Newton-Raphson and one explicit method. Finally, we exhibit some numerical simulations supporting our theoretical results. To the best of our knowledge, this is the first monotone finite difference discretization of the variational $p$-Laplacian and also the first time that nonhomogeneous problems for this operator can be treated numerically with a finite difference scheme.

Keywords $p$-Laplacian $\cdot$ Finite difference $\cdot$ Mean value property $\cdot$ Nonhomogeneous Dirichlet problem $\cdot$ Viscosity solutions $\cdot$ Dynamic programming principle

Mathematics Subject Classification $65 \mathrm{~N} 06 \cdot 35 \mathrm{~J} 60 \cdot 35 \mathrm{~J} 70 \cdot 35 \mathrm{~J} 75 \cdot 35 \mathrm{~J} 92 \cdot 35 \mathrm{D} 40 \cdot 35 \mathrm{~B} 05$

\section{Introduction and Main Results}

In the recent paper [10], we studied a new ${ }^{1}$ mean value formula (MVF) for the variational $p$-Laplace operator,

$$
\Delta_{p} u=\operatorname{div}\left(|\nabla u|^{p-2} \nabla u\right) .
$$

With the notation $J_{p}(t):=|t|^{p-2} t$ for all $p>1$, the MVF, valid for any $C^{2}\left(\mathbb{R}^{d}\right)$ function, reads

$$
\frac{1}{D_{d, p} r^{p}} f_{B_{r}} J_{p}(u(x+y)-u(x)) \mathrm{d} y=\Delta_{p} u(x)+o_{r}(1) \quad \text { as } \quad r \rightarrow 0^{+} .
$$

\section{Félix del Teso}

felix.delteso@uam.es

Erik Lindgren

erik.lindgren@math.uu.se

1 Departamento de Matemáticas, Universidad Autónoma de Madrid, Campus de Cantoblanco, Madrid 28049, Spain

2 Department of Mathematics, Uppsala University, Uppsala 480751 06, Sweden

1 This mean value formula was independently derived for $p \geq 2$ in [6], see Proposition 2.10 and Theorem 2.12 therein. 
Here $D_{d, p}:=\frac{d}{2(d+p)} f_{\partial B_{1}}\left|y_{1}\right|^{p} \mathrm{~d} \sigma(y)$, where $y_{1}$ is the first coordinate, $\mathrm{d} \sigma$ the surface measure on the sphere and $B_{r}$ denotes the ball of radius $r>0$ centered at 0 .

The aim of this paper is to propose a new monotone finite difference discretization of the $p$-Laplacian based on the asymptotic expansion (1.2). As an application of our discretization we also propose a convergent numerical scheme associated to the nonhomogeneous Dirichlet problem

$$
\begin{aligned}
-\Delta_{p} u(x) & =f(x), \quad x \in \Omega, \\
u(x) & =g(x), \quad x \in \partial \Omega .
\end{aligned}
$$

The scheme results in a nonlinear system. We propose two methods to solve this system: (1) Newton-Raphson and (2) an explicit method, based on the convergence to a steady state of an evolution problem. We comment the advantages of each one in Sect. 5. Finally, we exhibit some numerical tests of the accuracy and convergence of the scheme.

To the best of our knowledge, this is the first monotone finite difference discretization of the variational $p$-Laplacian available in the literature and therefore the first time that nonhomogeneous problems of the form (1.3)-(1.4) can be treated numerically via finite difference schemes. The monotonicity property (see Lemma 4.4) is crucial for the convergence of finite difference schemes in the context of viscosity solutions (see [4]). It is also worth mentioning that, in contrast to the finite difference schemes for the normalized (or game theoretical) $p$ Laplacian considered earlier (see Sect. 1.2), our scheme is well suited for Newton-Raphson solvers, which is an advantage when it comes to solving a nonlinear system effectively.

\subsection{Main Results}

In order to describe our main results we need to introduce some notation. Given a discretization parameter $h>0$, consider the uniform grid defined by $\mathcal{G}_{h}:=h \mathbb{Z}^{d}=\left\{y_{\alpha}:=h \alpha: \alpha \in\right.$ $\mathbb{Z}^{d}$ \}. Let $r>0$ and consider the following discrete operator

$$
\Delta_{p}^{h} \phi(x):=\frac{h^{d}}{D_{d, p} \omega_{d} r^{p+d}} \sum_{y_{\alpha} \in B_{r}} J_{p}\left(\phi\left(x+y_{\alpha}\right)-\phi(x)\right),
$$

where $\omega_{d}$ denotes the measure of the unit ball in $\mathbb{R}^{d}$. Throughout the paper, we will assume the following relation between $h$ and $r$ :

$$
h= \begin{cases}o\left(r^{\frac{p}{p-1}}\right), & \text { if } p \in(1,3) \backslash\{2\}, \\ o(r), & \text { if } p=2, \\ o\left(r^{\frac{3}{2}}\right), & \text { if } p \in[3, \infty) .\end{cases}
$$

Our first result regards the consistency of the discretization (1.5).

Theorem 1.1 Let $p \in(1, \infty), x \in \mathbb{R}^{d}$ and $\phi \in C^{2}\left(B_{R}(x)\right)$ for some $R>0$. Assume $(\mathrm{H})$. Then

$$
\Delta_{p}^{h} \phi(x)=\Delta_{p} \phi(x)+o_{r}(1) \text { as } r \rightarrow 0^{+} .
$$

Our second result concerns the finite difference numerical scheme for (1.3)-(1.4) induced by the discretization (1.5). More precisely, fix $r_{0}>0$ and let $\partial \Omega_{r}:=\left\{x \in \Omega^{c}: \operatorname{dist}(x, \Omega) \leq\right.$ $r\}, \Omega_{r}=\Omega \cup \partial \Omega_{r}$ and $G$ be a continuous extension of $g$ from $\partial \Omega$ to $\partial \Omega_{r}$ for all $r<r_{0}$. Consider $u_{h}: \Omega_{r} \rightarrow \mathbb{R}$ such that

$$
-\Delta_{p}^{h} u_{h}(x)=f(x), \quad x \in \Omega,
$$




$$
u_{h}(x)=G(x), \quad x \in \partial \Omega_{r} .
$$

We have the following result.

Theorem 1.2 Let $p \in(1, \infty), \Omega \subset \mathbb{R}^{d}$ be a bounded, open and $C^{2}$ domain, $f \in C(\bar{\Omega})$ and $g \in C(\partial \Omega)$. Assume $(\mathrm{H})$.

(a) Then there exists a unique pointwise solution $u_{h} \in L^{\infty}\left(\Omega_{r}\right)$ of (1.6)-(1.7) when $r$ is small enough.

(b) If $u$ is the unique viscosity solution of (1.3)-(1.4), then

$$
\sup _{x \in \bar{\Omega}}\left|u_{h}(x)-u(x)\right| \rightarrow 0 \text { as } r \rightarrow 0^{+} \text {. }
$$

Remark 1.3 We conjecture that the relation $h=o\left(r^{3 / 2}\right)$ is sufficient also in the range $p \in$ $(1,3)$. See Sect. 6.5 for numerical evidence supporting this.

We note that if we restrict (1.6)-(1.7) to the uniform grid $\mathcal{G}_{h}$ we obtain a fully discrete problem suited for numerical computations. More precisely, define the discrete sets

$$
B_{r}^{h}:=B_{r} \cap \mathcal{G}_{h}, \quad \Omega^{h}:=\Omega \cap \mathcal{G}_{h}, \quad \partial \Omega^{h}:=\partial \Omega \cap \mathcal{G}_{h} \quad \text { and } \quad \Omega_{r}^{h}:=\Omega_{r} \cap \mathcal{G}_{h} .
$$

Observe that $\Delta_{p}^{h}$ given in $(1.5)$ can be interpreted as an operator $\Delta_{p}^{h}: \ell^{\infty}\left(\mathcal{G}_{h}\right) \rightarrow \ell^{\infty}\left(\mathcal{G}_{h}\right)$ since given any $x_{\beta}, y_{\alpha} \in \mathcal{G}_{h}$ we have $x_{\beta}+y_{\alpha}=(\beta+\alpha) h=x_{\beta+\alpha} \in \mathcal{G}_{h}$ and then

$$
\Delta_{p}^{h} \phi_{\beta}:=\frac{h^{d}}{D_{d, p} \omega_{d} r^{p+d}} \sum_{y_{\alpha} \in B_{r}} J_{p}\left(\phi_{\beta+\alpha}-\phi_{\beta}\right) \text { for } x_{\beta} \in \Omega^{h}
$$

with $\phi: \mathcal{G}_{h} \rightarrow \mathbb{R}$ and $\phi_{\gamma}:=\phi(\gamma h)$, whenever $\gamma h \in \mathcal{G}_{h}$. Finally note that if $x_{\beta} \in \Omega^{h}$ and $y_{\alpha} \in B_{r}^{h}$ we have that $x_{\beta}+y_{\alpha}=x_{\beta+\alpha} \in \Omega_{r}^{h}$, so that (1.6)-(1.7) can be interpreted as

$$
\begin{aligned}
-\Delta_{p}^{h} U_{\beta} & =f_{\beta}, \quad x_{\beta} \in \Omega^{h} \\
U_{\beta} & =G_{\beta}, \quad x_{\beta} \in \partial \Omega_{r}^{h},
\end{aligned}
$$

with $U: \Omega_{r}^{h} \rightarrow \mathbb{R}, f_{\beta}:=f\left(x_{\beta}\right)$ and $G_{\beta}:=G\left(x_{\beta}\right)$. In this way we have the following trivial consequence of Theorem 1.2.

Corollary 1.4 Assume the hypotheses of Theorem 1.2.

(a) Then there exists a unique pointwise solution $U \in \ell^{\infty}\left(\Omega_{r}^{h}\right)$ of (1.8)-(1.9) when $r$ is small enough.

(b) If $u$ is the unique viscosity solution of (1.3)-(1.4), then

$$
\max _{x_{\beta} \in \Omega_{h}}\left|U_{\beta}-u\left(x_{\beta}\right)\right| \rightarrow 0 \text { as } r \rightarrow 0 .
$$

\subsection{Related Results}

For an overview of classical and modern results for the $p$-Laplacian, we refer the reader to the book [22]. For an overview of numerical methods for degenerate elliptic PDEs we refer the reader to Section 1.1 in [34].

We want to stress that the operator of interest in this paper is the variational $p$-Laplacian, i.e.,

$$
\Delta_{p} u=\operatorname{div}\left(|\nabla u|^{p-2} \nabla u\right)
$$


Once we have found a monotone discretization of $\Delta_{p}$, it is straightforward to find monotone finite difference schemes also for $p$-Laplace equations involving gradient terms, such as

$$
-\Delta_{p} u=|\nabla u|^{q_{1}} f(x)+|\nabla u|^{q_{2}},
$$

or other Hamilton-Jacobi-type equations involving the $p$-Laplacian, which do not necessarily allow for a variational formulation. In particular, we could recover and treat equations involving the normalized $p$-Laplacian (see (1.10)).

On the other hand, finite difference methods for equations involving the $p$-Laplacian have been successfully developed using the normalized (or game theoretical) version of the $p$-Laplacian $\Delta_{p}^{\mathrm{N}}$. The ideas are based on the identity

$$
\Delta_{p} u=|\nabla u|^{p-2} \Delta u+(p-2)|\nabla u|^{p-4} \Delta_{\infty} u .
$$

This allows to define

$$
\Delta_{p}^{\mathrm{N}} u:=|\nabla u|^{2-p} \Delta_{p} u=\Delta u+(p-2) \Delta_{\infty}^{\mathrm{N}} u,
$$

where $\Delta_{\infty}^{\mathrm{N}}$ is the so-called normalized infinity Laplacian, which is given by the second order directional derivative in the direction of the gradient. One limitation of such methods is the fact that they are not well adapted to treat nonhomogeneous problems of the form $-\Delta_{p} u=f$, unless $p \leq 2$. Instead they allow for treating inhomogeneities of the form $-\Delta_{p} u=|\nabla u|^{p-2} f$ (this problem is equivalent to $-\Delta_{p}^{\mathrm{N}} u=f$ ), which our method could handle as well, at least if $p \geq 2$ (since monotone approximations of $|\nabla u|$ are well known). Of course, both problems are equivalent only if $f \equiv 0$.

Let us first comment on the literature related to finite difference methods for $\Delta_{p}^{\mathrm{N}}$. In [34], the author presents a monotone finite difference scheme for the normalized infinity Laplacian and the game theoretical (or normalized) $p$-Laplacian for $p \geq 2$. In addition, a scheme for (1.3)-(1.4) with $f \equiv 0$ is presented, together with a semi-implicit solver. In [11], a strategy to prove the convergence of dynamic programming principles (including monotone finite difference schemes) for the normalized $p$-Laplacian is presented, as well as the strong uniqueness property for the $p$-Laplacian, which is crucial for the application of the convergence criteria of Barles and Souganidis in [4]. We also seize the opportunity mention Section 6 in [7], where a finite difference method (based on the mean value properties of the normalized $p$-Laplacian) is proposed for a double-obstacle problem involving the $p$ Laplacian. We note that in the case $1<p<2$ neither of the above mentioned schemes are monotone, and as such, the numerical scheme in this paper is the first one treating this range, even in the homogeneous case $f \equiv 0$.

There are many other monotone approximations of $\Delta_{p}^{\mathrm{N}}$ available in the literature. Strictly speaking, they are not numerical approximations, but the proof of convergence follows similar strategies based on monotonicity and consistency. See [11] for a discussion on this topic. Such approximations were first presented in [29] (see also [20,30,31] for a probabilistic game theoretical approach). The basic idea of these approximations is to combine the classical mean value property (MVP) for the Laplacian with a MVP for the normalized infinity Laplacian motivated by Tug-of-War games [35]. The literature on this topic has become extensive in the last decade. In $[2,23]$ the equivalence between being $p$-harmonic and satisfying a MVP is treated. See [16,19] for a MVP in the full range $1<p<\infty$ and [21] for the application of such approximations in the context of obstacle problems.

Regarding monotone approximations of the variational $p$-Laplacian, the literature is very recent and not so extensive. The MVP given by (1.2) was derived in [6,10]. In [10] it is shown 
to be a monotone approximation of $\Delta_{p}$. The authors are also able to prove convergence of the corresponding approximating problems to a viscosity solution.

It is noteworthy that the discretization presented in this paper is reminiscent of the definition of the variational $p$-Laplacian on graphs, see [1] and also [37]. In this direction, Corollary 1.4 can be interpreted as the convergence of the solution to a PDE defined on a graph associated to the grid. We refer to the recent paper [36] for a study of the eigenvalues of this operator and to [12] for its applications to image processing. Note that also the normalized $p$-Laplacian has been defined on graphs, see [28].

Finally, we seize the opportunity to mention that since the $p$-Laplacian is of divergence form, it is well suited for finite element based methods. We mention a few papers in this direction: [5,13,14,17,24-27]. We want to stress that finite element methods are not well suited for being treated with viscosity methods.

\subsection{Organization of the Paper}

In Sect. 2, we introduce some notation and prerequisites needed in the rest of the paper. Section 3 is devoted to the proof of consistency of the discretization previously introduced. In Sect. 4, we study the numerical scheme for the boundary value problems. This is followed by a discussion around solving the nonlinear systems of equations derived from our scheme, in Sect. 5. Finally, in Sect. 6, we perform some numerical experiments to support our theoretical results. We also have an appendix containing technical results and a discussion regarding the invertibility of the Jacobian used in one of the methods in Sect. 5.

\section{Notations and Prerequisites}

We adopt the following definition of viscosity solutions, which is the classical definition adjusted to the nonhomogeneous equation (see e.g. [15]).

Definition 2.1 (Solutions of the equation) Suppose that $f \in C(\Omega)$. We say that a lower (resp. upper) semicontinuous function $u$ in $\Omega$ is a viscosity supersolution (resp. subsolution) of the equation

$$
-\Delta_{p} u=f
$$

in $\Omega$ if the following holds: whenever $x_{0} \in \Omega$ and $\varphi \in C^{2}\left(B_{R}\left(x_{0}\right)\right)$ for some $R>0$ are such that $|\nabla \varphi(x)| \neq 0$ for $x \in B_{R}\left(x_{0}\right) \backslash\left\{x_{0}\right\}$,

$$
\varphi\left(x_{0}\right)=u\left(x_{0}\right) \text { and } \varphi(x) \leq u(x) \quad(\operatorname{resp} \cdot \varphi(x) \geq u(x)) \text { for all } x \in B_{R}\left(x_{0}\right) \cap \Omega,
$$

then we have

$$
\lim _{\rho \rightarrow 0} \sup _{B_{\rho}\left(x_{0}\right) \backslash\left\{x_{0}\right\}}\left(-\Delta_{p} \varphi(x)\right) \geq f\left(x_{0}\right) \quad\left(\text { resp. } \lim _{\rho \rightarrow 0} \inf _{B_{\rho}\left(x_{0}\right) \backslash\left\{x_{0}\right\}}\left(-\Delta_{p} \varphi(x)\right) \leq f\left(x_{0}\right)\right) .
$$

A viscosity solution is a function $u \in C(\Omega)$ being both a viscosity supersolution and a viscosity subsolution.

Remark 2.1 We consider condition (2.1) to avoid problems with the definition of $-\Delta_{p} \varphi\left(x_{0}\right)$ when $\left|\nabla \varphi\left(x_{0}\right)\right|=0$ and $p \in(1,2)$. However, when either $p \geq 2$ or $\left|\nabla \varphi\left(x_{0}\right)\right| \neq 0$, (2.1) can be replaced by the standard one, i.e.,

$$
-\Delta_{p} \varphi\left(x_{0}\right) \geq f\left(x_{0}\right) \quad\left(\text { resp. }-\Delta_{p} \varphi\left(x_{0}\right) \leq f\left(x_{0}\right)\right) .
$$


A viscosity solution of the boundary value problem (1.3)-(1.4) attaining the boundary condition in a pointwise sense is naturally defined as follows.

Definition 2.2 (Solutions of the boundary value problem) Suppose that $f \in C(\bar{\Omega})$ and $g \in$ $C(\partial \Omega)$. We say that a lower (resp. upper) semicontinuous function $u$ in $\bar{\Omega}$ is a viscosity supersolution (resp. subsolution) of (1.3)-(1.4) if

(a) $u$ is a viscosity supersolution (resp. subsolution) of $-\Delta_{p} u=f$ in $\Omega$ (as in Definition 2.1);

(b) $u(x) \geq g(x)(\operatorname{resp} . u(x) \leq g(x))$ for $x \in \partial \Omega$.

A viscosity solution of (1.3)-(1.4) is a function $u \in C(\bar{\Omega})$ being both a viscosity supersolution and a viscosity subsolution.

Remark 2.2 To prove the convergence result (Theorem 1.2(b)) we will make use of a generalized notion of viscosity solutions of a boundary value problem. We will introduce this notion just before using it. See Sect. 4.3.

\section{Consistency of the Discretization: Proof of Theorem 1.1}

In this section we prove the consistency of the discretization $\Delta_{p}^{h}$ for $C^{2}$-functions as presented in Theorem 1.1.

Proof of Theorem 1.1 Throughout this proof, $C$ will denote a constant that may depend on $p$, the dimension $d$, but not on $r$ or $h$.

The mean value property introduced in [10] involves the quantity

$$
\mathcal{M}_{r}^{p}[\phi](x)=\frac{1}{D_{d, p} r^{p}} f_{B_{r}} J_{p}(\phi(x+y)-\phi(x)) \mathrm{d} y .
$$

By the triangle inequality and Theorem 2.1 in [10]

$$
\begin{aligned}
\left|\Delta_{p}^{h} \phi(x)-\Delta_{p} \phi(x)\right| & \leq\left|\Delta_{p}^{h} \phi(x)-\mathcal{M}_{r}^{p}[\phi](x)\right|+\left|\mathcal{M}_{r}^{p}[\phi](x)-\Delta_{p} \phi(x)\right| \\
& =\left|\Delta_{p}^{h} \phi(x)-\mathcal{M}_{r}^{p}[\phi](x)\right|+o_{r}(1) \quad \text { as } \quad r \rightarrow 0^{+} .
\end{aligned}
$$

Therefore, it is sufficient to show that

$$
\left|\Delta_{p}^{h} \phi(x)-\mathcal{M}_{r}^{p}[\phi](x)\right|=o_{r}(1) \quad \text { as } \quad r \rightarrow 0^{+} .
$$

Step 1: Approximation of $B_{r}$ by $h$-boxes. Define the following family of $h$-boxes centred at $y_{\alpha} \in \mathcal{G}_{h}$,

$$
R_{\alpha}^{h}:=y_{\alpha}+\frac{h}{2}[-1,1)^{d},
$$

and the union of boxes that approximates $B_{r}$

$$
\tilde{B}_{r}:=\bigcup_{y_{\alpha} \in B_{r}^{h}} R_{\alpha}^{h}
$$




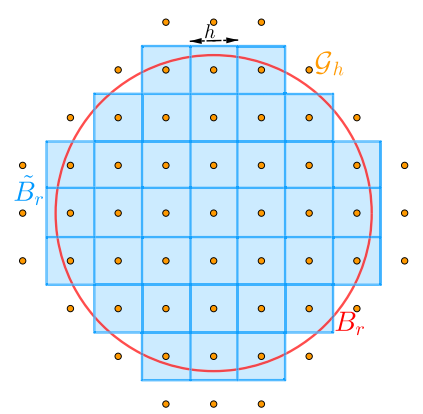

Fig. 1 The family of boxes and their union $\tilde{B}_{r}$ that covers $B_{r}$

See Fig. 1.

Consider

$$
A_{r}:=\frac{1}{2} \int_{B_{r}}\left(J_{p}(\phi(x+y)-\phi(x))+J_{p}(\phi(x-y)-\phi(x))\right) \mathrm{d} y,
$$

and

$$
\tilde{A}_{r}:=\frac{1}{2} \int_{\tilde{B}_{r}}\left(J_{p}(\phi(x+y)-\phi(x))+J_{p}(\phi(x-y)-\phi(x))\right) \mathrm{d} y .
$$

In this step we will prove that

$$
\left|A_{r}-\tilde{A}_{r}\right|=o\left(r^{d+p}\right)
$$

Notice first that

$$
\begin{aligned}
\left|A_{r}-\tilde{A}_{r}\right|= & \frac{1}{2} \mid \int_{B_{r} \backslash \tilde{B}_{r}}\left(J_{p}(\phi(x+y)-\phi(x))+J_{p}(\phi(x-y)-\phi(x))\right) \mathrm{d} y \\
& \quad-\int_{\tilde{B}_{r} \backslash B_{r}}\left(J_{p}(\phi(x+y)-\phi(x))+J_{p}(\phi(x-y)-\phi(x))\right) \mathrm{d} y \mid \\
\leq & \frac{1}{2} \int_{\left(B_{r} \cup \tilde{B}_{r}\right) \backslash\left(B_{r} \cap \tilde{B}_{r}\right)}\left|J_{p}(\phi(x+y)-\phi(x))+J_{p}(\phi(x-y)-\phi(x))\right| \mathrm{d} y .
\end{aligned}
$$

It is easy to verify that $B_{r} \cup \tilde{B}_{r} \subset B_{r+\sqrt{d} h}$ and $B_{r-\sqrt{d} h} \subset B_{r} \cap \tilde{B}_{r}$ so that

$$
\left(B_{r} \cup \tilde{B}_{r}\right) \backslash\left(B_{r} \cap \tilde{B}_{r}\right) \subset B_{r+\sqrt{d} h} \backslash B_{r-\sqrt{d} h} .
$$

Observe that regardless of the value of $p$, we always have $h=o(r)$. Therefore,

$$
\begin{aligned}
& \left|\left(B_{r} \cup \tilde{B}_{r}\right) \backslash\left(B_{r} \cap \tilde{B}_{r}\right)\right| \leq\left|B_{r+\sqrt{d} h} \backslash B_{r-\sqrt{d} h}\right|=\omega_{d}\left((r+\sqrt{d} h)^{d}-(r-\sqrt{d} h)^{d}\right) \\
& \leq \omega_{d} d(r+\sqrt{d} h)^{d-1} 2 \sqrt{d} h \leq C r^{d-1} h \\
& =o\left(r^{d}\right) \text {. }
\end{aligned}
$$

On the other hand, by Taylor expansion

$$
|\phi(x+y)-\phi(x)+\phi(x-y)-\phi(x)|=\mathcal{O}\left(|y|^{2}\right) .
$$


In the case $p \geq 2$, Lemma A.1 implies

$$
\begin{aligned}
& \left|J_{p}(\phi(x+y)-\phi(x))+J_{p}(\phi(x-y)-\phi(x))\right| \\
& \quad=\left|J_{p}(\phi(x+y)-\phi(x))-J_{p}(-\phi(x-y)+\phi(x))\right| \\
& \leq C \max (|\phi(x-y)-\phi(x)|,|\phi(x+y)-\phi(x)|)^{p-2} \mid \phi(x+y) \\
& \quad-\phi(x)+\phi(x-y)-\phi(x) \mid \\
& =O\left(|y|^{p}\right) .
\end{aligned}
$$

We can conclude

$$
\begin{aligned}
\left|A_{r}-\tilde{A}_{r}\right| & \leq \frac{\tilde{C}}{2} \int_{\left(B_{r} \cup \tilde{B}_{r}\right) \backslash\left(B_{r} \cap \tilde{B}_{r}\right)}|y|^{p} \mathrm{~d} y \leq \frac{1}{2}(r+\sqrt{d} h)^{p}\left|\left(B_{r} \cup \tilde{B}_{r}\right) \backslash\left(B_{r} \cap \tilde{B}_{r}\right)\right| \\
& =o\left(r^{p+d}\right) .
\end{aligned}
$$

In the case $p<2$, we argue slightly different. On page 8 in [10] it is proved that

$$
f_{\partial B_{r}}\left|J_{p}(\phi(x+y)-\phi(x))-J_{p}\left(y \cdot \nabla \phi(x)+\frac{1}{2} y^{T} D^{2} \phi(x) y\right)\right| d y=o\left(r^{p}\right) .
$$

In a similar fashion, one can prove

$$
\begin{aligned}
\int_{\left(B_{r} \cup \tilde{B}_{r}\right) \backslash\left(B_{r} \cap \tilde{B}_{r}\right)} \mid & J_{p}(\phi(x+y)-\phi(x))+J_{p}(\phi(x-y)-\phi(x)) \\
& -J_{p}\left(y \cdot \nabla \phi(x)+\frac{1}{2} y^{T} D^{2} \phi(x) y\right)-J_{p}(-y \cdot \nabla \phi(x) \\
& \left.+\frac{1}{2} y^{T} D^{2} \phi(x) y\right) \mid d y=o\left(r^{p+d}\right) .
\end{aligned}
$$

To show (3.1) it is therefore sufficient to show that

$$
\begin{aligned}
& \int_{\left(B_{r} \cup \tilde{B}_{r}\right) \backslash\left(B_{r} \cap \tilde{B}_{r}\right)} \mid J_{p}\left(y \cdot \nabla \phi(x)+\frac{1}{2} y^{T} D^{2} \phi(x) y\right)+J_{p}(-y \cdot \nabla \phi(x) \\
& \left.+\frac{1}{2} y^{T} D^{2} \phi(x) y\right) \mid d y=o\left(r^{p+d}\right) .
\end{aligned}
$$

Without loss of generality assume that $\nabla \phi(x)=c e_{1}$ with $c \neq 0$. Then

$$
\begin{aligned}
J_{p}\left(y \cdot \nabla \phi(x)+\frac{1}{2} y^{T} D^{2} \phi(x) y\right) & =J_{p}\left(c y \cdot \mathbf{e}_{1}+\frac{1}{2} y^{T} D^{2} \phi(x) y\right)=(c|y|)^{p-1} J_{p}\left(\hat{y} \cdot \mathbf{e}_{1}\right. \\
& \left.+\frac{1}{2} c^{-1}|y| \hat{y}^{T} D^{2} \phi(x) \hat{y}\right)
\end{aligned}
$$

where $\hat{y}=y /|y|$. By Lemma A.2 with $a=\hat{y} \cdot \mathbf{e}_{1}$ and $b=\frac{1}{2} c^{-1}|y| \hat{y}^{T} D^{2} \phi(x) \hat{y}$ we get

$$
\begin{aligned}
& (c|y|)^{p-1}\left|J_{p}\left(\hat{y} \cdot \mathbf{e}_{1}+\frac{1}{2} c^{-1}|y| \hat{y}^{T} D^{2} \phi(x) \hat{y}\right)-J_{p}\left(\hat{y} \cdot \mathbf{e}_{1}\right)\right| \\
& \quad \leq C(c|y|)^{p-1}\left(\left|\hat{y} \cdot \mathbf{e}_{1}\right|+\frac{1}{2} c^{-1}|y|\left|\hat{y}^{T} D^{2} \phi(x) \hat{y}\right|\right)^{p-2} \frac{1}{2} c^{-1}|y|\left|\hat{y}^{T} D^{2} \phi(x) \hat{y}\right| \\
& \leq C|y|^{p}\left|\hat{y} \cdot \mathbf{e}_{1}\right|^{p-2} .
\end{aligned}
$$

Hence,

$\left|J_{p}\left(y \cdot \nabla \phi(x)+\frac{1}{2} y^{T} D^{2} \phi(x) y\right)+J_{p}\left(-y \cdot \nabla \phi(x)+\frac{1}{2} y^{T} D^{2} \phi(x) y\right)\right| \leq C|y|^{p}\left|\hat{y} \cdot \mathbf{e}_{1}\right|^{p-2}$. 
From (6.2) in [10] it then follows that

$$
\begin{gathered}
\int_{\partial B_{r}}\left|J_{p}\left(y \cdot \nabla \phi(x)+\frac{1}{2} y^{T} D^{2} \phi(x) y\right)+J_{p}\left(-y \cdot \nabla \phi(x)+\frac{1}{2} y^{T} D^{2} \phi(x) y\right)\right| d \sigma(y) \\
\leq C r^{p} \int_{\partial B_{r}}\left|\hat{y} \cdot \mathbf{e}_{1}\right|^{p-2} \mathrm{~d} \sigma(y) \leq \tilde{C} r^{p+d-1} .
\end{gathered}
$$

After integration (to pass from spheres to balls) we obtain

$$
\begin{aligned}
\int_{\left(B_{r} \cup \tilde{B}_{r}\right) \backslash\left(B_{r} \cap \tilde{B}_{r}\right)} \mid & J_{p}\left(y \cdot \nabla \phi(x)+\frac{1}{2} y^{T} D^{2} \phi(x) y\right)+J_{p}\left(-y \cdot \nabla \phi(x)+\frac{1}{2} y^{T} D^{2} \phi(x) y\right) \mid d y \\
\leq & \int_{B_{r+\sqrt{d} h} \backslash B_{r-\sqrt{d} h}} \mid J_{p}\left(y \cdot \nabla \phi(x)+\frac{1}{2} y^{T} D^{2} \phi(x) y\right)+J_{p}(-y \cdot \nabla \phi(x) \\
& \left.+\frac{1}{2} y^{T} D^{2} \phi(x) y\right) \mid d y \\
\leq & C h r^{p+d-1}=o\left(r^{d+p}\right) .
\end{aligned}
$$

This is (3.2).

Step 2: Discretization of $\tilde{A}_{r}$. Consider

$$
\tilde{A}_{r}^{h}:=h^{d} \sum_{y_{\alpha} \in B_{r}} J_{p}\left(\phi\left(x+y_{\alpha}\right)-\phi(x)\right) .
$$

We will show that

$$
\left|\tilde{A}_{r}-\tilde{A}_{r}^{h}\right|=o\left(r^{d+p}\right)
$$

Observe that

$$
\begin{aligned}
\tilde{A}_{r} & =\frac{1}{2} \sum_{y_{\alpha} \in B_{r}} \int_{R_{\alpha}^{h}}\left(J_{p}(\phi(x+y)-\phi(x))+J_{p}(\phi(x-y)-\phi(x))\right) \mathrm{d} y \\
& =\frac{1}{2} \sum_{y_{\alpha} \in B_{r}} \int_{R_{0}^{h}}\left(J_{p}\left(\phi\left(x+y_{\alpha}+y\right)-\phi(x)\right)+J_{p}\left(\phi\left(x+y_{\alpha}-y\right)-\phi(x)\right)\right) \mathrm{d} y .
\end{aligned}
$$

Since $\left|R_{0}^{h}\right|=h^{d}$ we have

$$
\begin{aligned}
\mid \tilde{A}_{r}- & A_{r}^{h} \mid \\
= & \frac{1}{2} \mid \sum_{y_{\alpha} \in B_{r}} \int_{R_{0}^{h}}\left(J_{p}\left(\phi\left(x+y_{\alpha}+y\right)-\phi(x)\right)+J_{p}\left(\phi\left(x+y_{\alpha}-y\right)-\phi(x)\right)\right. \\
& \left.\quad-2 J_{p}\left(\phi\left(x+y_{\alpha}\right)-\phi(x)\right)\right) \mathrm{d} y \mid .
\end{aligned}
$$

If $p \geq 2$ we use Taylor expansion of order two and obtain

$$
\phi\left(x+y_{\alpha} \pm y\right)-\phi(x)=\phi\left(x+y_{\alpha}\right)-\phi(x) \pm \nabla \phi\left(x+y_{\alpha}\right) \cdot y+\mathcal{O}\left(y^{2}\right) .
$$

Let $\rho=\phi\left(x+y_{\alpha}\right)-\phi(x)$ and $\eta=\nabla \phi\left(x+y_{\alpha}\right)$. Then this can be expressed as

$$
\phi\left(x+y_{\alpha}+y\right)-\phi(x)=\rho+\eta \cdot y+\mathcal{O}\left(|y|^{2}\right) .
$$


Therefore, by Lemma A.1

$$
\begin{aligned}
& \left|J_{p}\left(\phi\left(x+y_{\alpha}+y\right)-\phi(x)\right)-J_{p}(\rho+\eta \cdot y)\right| \\
& \quad \leq C \max \left(\left|\phi\left(x+y_{\alpha}+y\right)-\phi(x)\right|,|\rho+\eta \cdot y|\right)^{p-2}|y|^{2} \\
& \quad \leq C r^{p-2} o\left(r^{2}\right) \\
& \quad=o\left(r^{p}\right),
\end{aligned}
$$

where we have used that $y=\mathcal{O}(h)=o(r)$ and that $\rho=\mathcal{O}\left(y_{\alpha}\right)=\mathcal{O}(r)$. It follows that it will be enough to obtain an estimate of the form

$$
\left|J_{p}(\rho+\eta \cdot y)+J_{p}(\rho-\eta \cdot y)-2 J_{p}(\rho)\right|=o\left(r^{p}\right) .
$$

For $p=2$, this estimate is trivial. When $p>3$ we use the second order Taylor expansion of $J_{p}$ to obtain

$$
\begin{aligned}
\left.\left|J_{p}(\rho+\eta \cdot y)-J_{p}(\rho)-(p-1)\right| \rho\right|^{p-2} \eta \cdot y \mid & \leq C \max (|\rho|,|\rho+\eta \cdot y|)^{p-3}|\eta \cdot y|^{2} \\
& \leq C r^{p-3} o\left(r^{3}\right)=o\left(r^{p}\right),
\end{aligned}
$$

since $\rho=\mathcal{O}\left(y_{\alpha}\right)=\mathcal{O}(r)$ and $y=\mathcal{O}(h)=o\left(r^{\frac{3}{2}}\right)$ when $p>3$.

When $p \in(2,3]$ we use the fact that the derivative of the function $t \mapsto J_{p}(t)$ is $(p-2)$ Hölder continuous and obtain

$$
\begin{aligned}
\left.\left|J_{p}(\rho+\eta \cdot y)-J_{p}(\rho)-(p-1)\right| \rho\right|^{p-2} \eta \cdot y \mid & \leq C|\eta \cdot y|^{p-1} \\
& =o\left(r^{p}\right)
\end{aligned}
$$

where we used that $y=\mathcal{O}(h)=o\left(r^{p /(p-1)}\right)$ when $p \in(2,3]$. The estimate (3.4) follows immediately from (3.5) and (3.6), respectively.

If $p<2$ we use the fact that $J_{p}$ is $(p-1)$-Hölder continuous. Thus,

$$
\begin{aligned}
\left|J_{p}\left(\phi\left(x+y_{\alpha}+y\right)-\phi(x)\right)-J_{p}\left(\phi\left(x+y_{\alpha}\right)-\phi(x)\right)\right| & \leq C\left|\phi\left(x+y_{\alpha}+y\right)-\phi\left(x+y_{\alpha}\right)\right|^{p-1} \\
& \leq C|y|^{p-1}=o\left(r^{p}\right),
\end{aligned}
$$

where we used the assumption $y=\mathcal{O}(h)=o\left(r^{p /(p-1)}\right)$ when $p<2$. Using (3.4) and (3.7) we get

$$
\left|\tilde{A}_{r}-A_{r}^{h}\right|=o\left(r^{p}\right) \sum_{y_{\alpha} \in B_{r}} h^{d}=o\left(r^{p}\right)\left|\tilde{B}_{r}\right| \leq o\left(r^{p}\right)\left|B_{r+\sqrt{d} h}\right|=o\left(r^{p+d}\right) .
$$

Step 3: Conclusion. Combining Step 1 and Step 2, we obtain

$$
\begin{aligned}
\left|\Delta_{p}^{h} \phi(x)-\mathcal{M}_{r}^{p}[\phi](x)\right| & =\frac{1}{D_{d, p^{r}}\left|B_{r}\right|}\left|A_{r}^{h}-A_{r}\right| \\
& \leq \frac{C}{r^{p+d}}\left(\left|A_{r}^{h}-\tilde{A}_{r}\right|+\left|\tilde{A}_{r}-A_{r}\right|\right)=\frac{1}{r^{p+d}} o\left(r^{p+d}\right)=o_{r}(1) .
\end{aligned}
$$

\section{Properties of the Numerical Scheme}

In this section we will state and prove some properties of the numerical scheme (1.6)-(1.7). 


\subsection{Existence and Uniqueness}

We will obtain the existence and uniqueness result given in Theorem 1.2(a).

First note that we can write

$$
\begin{aligned}
\Delta_{p}^{h} \phi(x) & =\frac{h^{d}}{D_{d, p} \omega_{d} r^{p+d}} \sum_{y_{\alpha} \in B_{r}} J_{p}\left(\phi\left(x+y_{\alpha}\right)-\phi(x)\right) \\
& =\frac{1}{D_{d, p} r^{p}} f_{B_{r}} J_{p}(\phi(x+y)-\phi(x)) \mathrm{d} \mu(y)
\end{aligned}
$$

with $\mu$ being the discrete measure given by

$$
\mathrm{d} \mu(y):=h^{d} \sum_{y_{\alpha} \in B_{r}} \mathrm{~d} \delta_{y_{\alpha}}(y),
$$

where $\delta_{z}$ denotes the dirac delta measure at $z \in \mathbb{R}^{d}$. With this simple observation, all the results of Section 9.1 in [10] follow here word by word (replacing $\mathcal{M}_{r}^{p}$ by $\Delta_{p}^{h}$ and $\mathrm{d} y$ by $\mathrm{d} \mu(y))$. We state them for completeness. Our running assumptions in this section will be $f \in C(\bar{\Omega})$ and $G \in C\left(\partial \Omega_{r}\right)$ (a continuous extension of $g \in C(\partial \Omega)$ ).

The comparison result below implies in particular the uniqueness of solutions of (1.6)(1.7).

Proposition 4.1 (Comparison) Let $p \in(1, \infty), h, r>0$, and $v, w \in L^{\infty}\left(\Omega_{r}\right)$ be such that

$$
\left\{\begin{array} { c l } 
{ - \Delta _ { p } ^ { h } w ( x ) \geq f ( x ) , } & { x \in \Omega , } \\
{ w ( x ) \geq G ( x ) , } & { x \in \partial \Omega _ { r } , }
\end{array} \quad \text { and } \quad \left\{\begin{array}{rl}
-\Delta_{p}^{h} v(x) \leq f(x), & x \in \Omega, \\
v(x) \leq G(x), & x \in \partial \Omega_{r} .
\end{array}\right.\right.
$$

Then $v \leq w$ in $\Omega_{r}$.

The existence of solutions is proved by a monotonicity argument. For this purpose, we need the following $L^{\infty}$-bound.

Proposition 4.2 ( $\mathrm{L}^{\infty}$-bound) Let $p \in(1, \infty)$, let $R>0$ and $u_{h}$ be the solution (if any) of (1.6)-(1.7) corresponding to some $r \leq R$. Assume $(\mathrm{H})$. Then

$$
\left\|u_{h}\right\|_{\infty} \leq A
$$

for $r$ small enough, with $A>0$ depending on $p, \Omega, f, g$ and $R$ (but not on $r$ and $h$ ).

Proof See the proof of Proposition 9.2 [10]. The proof is based on an explicit barrier for the p-Laplace equation, which by Theorem 1.1 gives a barrier for (1.6)-(1.7).

In order to prove the existence we also need a two step iteration process. For that purpose we define

$$
L[\psi, \phi](x):=\frac{1}{D_{d, p^{r}}{ }^{p}} f_{B_{r}} J_{p}(\phi(x+y)-\psi(x)) \mathrm{d} \mu(y) .
$$

We have the following result.

Lemma 4.3 Let $r>0$ and $\phi \in L^{\infty}\left(\Omega_{r}\right)$.

(a) Then there exists a unique $\psi \in L^{\infty}(\Omega)$ such that $-L[\psi, \phi](x)=f(x)$ for all $x \in \Omega$.

(b) Let $\psi_{1}$ and $\psi_{2}$ be such that $-L\left[\psi_{1}, \phi\right](x) \leq f(x)$ and $-L\left[\psi_{2}, \phi\right](x) \geq f(x)$ for all $x \in \Omega$, then $\psi_{1} \leq \psi_{2}$ in $\Omega$. 
Proof The proof follows as the proof of Lemma 9.3 in [10].

We are finally ready to prove the existence.

Proof of Theorem 1.2(a) The proof follows the proof of Proposition 9.4 in [10]. We spell out some details below.

The approach for existence is to construct a monotone increasing sequence converging to the solution. Let $\mathcal{B}$ be the barrier constructed in Proposition 4.2. Define

$$
u_{h}^{0}(x)= \begin{cases}\inf _{\partial \Omega_{r}} G-\mathcal{B}(x) & x \in \Omega, \\ G(x) & x \in \partial \Omega_{r},\end{cases}
$$

and the sequence $u_{h}^{k}$ as the sequence of solutions of

$$
\begin{cases}-L\left[u_{h}^{k}, u_{h}^{k-1}\right](x)=f(x) & x \in \Omega, \\ u_{h}^{k}(x)=G(x) & x \in \partial \Omega_{r} .\end{cases}
$$

One can prove that $u_{h}^{k}$ exists for all $k$, is nondecreasing (by the monotonicity of $L$ ) and uniformly bounded (by Proposition 4.2). We can then define the pointwise limit

$$
u_{h}(x):=\lim _{k \rightarrow \infty} u_{h}^{k}(x)
$$

Due to the the pointwise convergence

$$
-f(x)=\lim _{k \rightarrow \infty} L\left[u_{h}^{k+1}, u_{h}^{k}\right](x)=L\left[\lim _{k \rightarrow \infty} u_{h}^{k+1}, \lim _{k \rightarrow \infty} u_{h}^{k}\right](x)=L\left[u_{h}, u_{h}\right](x)=\Delta_{p}^{h}\left[u_{h}\right](x) .
$$

Thus, $u$ is a solution of (1.6). Clearly $u_{h}=G$ in $\partial \Omega_{r}$ so it is also a solution of (1.7). The uniqueness follows from Proposition 4.1.

\subsection{Monotonicity and Consistency}

In order to prove convergence of the numerical scheme, we will need certain monotonicity and consistency properties (we already obtained a uniform bound in Proposition 4.2). For a function $\phi: \Omega_{r} \rightarrow \mathbb{R}$ define

$S(r, h, x, \phi(x), \phi):= \begin{cases}-\frac{h^{d}}{D_{d, p} \omega_{d} r^{p+d}} \sum_{y_{\alpha} \in B_{r}} J_{p}\left(\phi\left(x+y_{\alpha}\right)-\phi(x)\right)-f(x) & x \in \Omega, \\ \phi(x)-G(x) & x \in \partial \Omega_{r} .\end{cases}$

Note that (1.6)-(1.7) can be equivalently formulated as

$$
S\left(r, h, x, u_{h}(x), u_{h}\right)=0 \quad x \in \Omega_{r} .
$$

We have the following result.

Lemma 4.4 Assume $(\mathrm{H})$.

(a) (Monotonicity) Let $t \in \mathbb{R}$ and $\psi \geq \phi$. Then

$$
S(r, h, x, t, \psi) \leq S(r, h, x, t, \phi)
$$


(b) (Consistency) For all $x \in \bar{\Omega}$ and $\phi \in C^{2}\left(B_{R}(x)\right)$ for some $R>0$ such that $|\nabla \phi(x)| \neq 0$ we have that

$$
\begin{aligned}
& \limsup _{r \rightarrow 0, z \rightarrow x, \xi \rightarrow 0} S\left(r, h, z, \phi(z)+\xi+\eta_{r}, \phi+\xi\right) \\
& \leq\left\{\begin{array}{cc}
-\Delta_{p} \phi(x)-f(x) & \text { if } x \in \Omega \\
\max \left\{-\Delta_{p} \phi(x)-f(x), \phi(x)-g(x)\right\} & \text { if } x \in \partial \Omega
\end{array}\right.
\end{aligned}
$$

and

$$
\begin{aligned}
\liminf _{r \rightarrow 0, z \rightarrow x, \xi \rightarrow 0} S\left(r, h, z, \phi(z)+\xi-\eta_{r}, \phi+\xi\right) & \\
\geq & \left\{\begin{array}{cc}
-\Delta_{p} \phi(x)-f(x) & \text { if } x \in \Omega \\
\min \left\{-\Delta_{p} \phi(x)-f(x), \phi(x)-g(x)\right\} & \text { if } x \in \partial \Omega,
\end{array}\right.
\end{aligned}
$$

where $0 \leq \eta_{r}=o\left(r^{p}\right)$ as $r \rightarrow 0^{+}$.

Proof The proof follows as in Lemma 9.7 in [10]. For part (b) it is essential to use the fact that $J_{p}$ is a Hölder continuous function, the basic properties of lim sup and lim inf and the consistency of $\Delta_{p}^{h}$ given in Theorem 1.1.

\subsection{Convergence}

We are now ready to prove the convergence stated in Theorem 1.2. The idea of the proof originates from [4]. The proof is almost the same as the proof of Theorem 2.5 ii) in [10]. We point out that it was necessary to adapt the proof in order to make it fit with the definition of viscosity solutions in the case $p \in(1,2)$. Below, we spell out some details.

First we need another definition of viscosity solutions of the boundary value problem and two auxiliary results that are taken from [10].

Definition 4.1 (Generalized viscosity solutions of the boundary value problem) Let $f \in$ $C(\bar{\Omega})$ and $g \in C(\partial \Omega)$. We say that a lower (resp. upper) semicontinuous function $u$ in $\bar{\Omega}$ is a generalized viscosity supersolution (resp. subsolution) of (1.3)-(1.4) in $\bar{\Omega}$ if whenever $x_{0} \in \bar{\Omega}$ and $\varphi \in C^{2}\left(B_{R}\left(x_{0}\right)\right)$ for some $R>0$ are such that $|\nabla \varphi(x)| \neq 0$ for $x \in B_{R}\left(x_{0}\right) \backslash\left\{x_{0}\right\}$,

$$
\varphi\left(x_{0}\right)=u\left(x_{0}\right) \text { and } \varphi(x) \leq u(x)(\operatorname{resp} \cdot \varphi(x) \geq u(x)) \text { for all } x \in B_{R}\left(x_{0}\right) \cap \bar{\Omega},
$$

then we have

$$
\begin{gathered}
\lim _{\rho \rightarrow 0} \sup _{B_{\rho\left(x_{0}\right) \backslash\left\{x_{0}\right\}}}\left(-\Delta_{p} \varphi(x)-f\left(x_{0}\right)\right) \geq 0 \text { if } x_{0} \in \Omega \\
\left(\text { resp. } \lim _{\rho \rightarrow 0} \inf _{B_{\rho\left(x_{0}\right)} \backslash\left\{x_{0}\right\}}\left(-\Delta_{p} \varphi(x)-f\left(x_{0}\right)\right) \leq 0\right) \\
\max \left\{\lim _{\rho \rightarrow 0} \sup _{\left.B_{\rho\left(x_{0}\right)}\right) \backslash\left\{x_{0}\right\}}\left(-\Delta_{p} \varphi(x)-f\left(x_{0}\right)\right), u\left(x_{0}\right)-g\left(x_{0}\right)\right\} \geq 0 \text { if } x_{0} \in \partial \Omega \\
\left(\text { resp. } \min \left\{\lim _{\rho \rightarrow 0} \inf _{B_{\rho\left(x_{0}\right)} \backslash\left\{x_{0}\right\}}\left(-\Delta_{p} \varphi(x)-f\left(x_{0}\right)\right), u\left(x_{0}\right)-g\left(x_{0}\right)\right\} \leq 0\right)
\end{gathered}
$$

Remark 4.5 As in Remark 2.1, we note that when either $p \geq 2$ or $\left|\nabla \varphi\left(x_{0}\right)\right| \neq 0$, the limits in the above definition can simply be replaced by $\left(-\Delta_{p} \varphi\left(x_{0}\right)-f\left(x_{0}\right)\right)$.

The following uniqueness result is Theorem 9.5 in [10]. 
Theorem 4.6 (Strong uniqueness property) Let $\Omega$ be a bounded $C^{2}$ domain. If $u$ and $v$ are generalized viscosity subsolutions and supersolutions of (1.3)-(1.4) respectively, then $u \leq v$.

We also need that a generalized viscosity solution is a (usual) viscosity solution in the case of a bounded $C^{2}$ domain. The proposition below is Proposition 9.6 in [10].

Proposition 4.7 Let $\Omega$ be a bounded $C^{2}$ domain. Then $u$ is a viscosity subsolution (resp. supersolution) of (1.3)-(1.4) if and only if $u$ is a generalized viscosity subsolution (resp. supersolution) of (1.3)-(1.4).

Proof of Theorem 1.2(b) Define

$$
\bar{u}(x)=\limsup _{r \rightarrow 0, y \rightarrow x} u_{h}(y), \quad \underline{u}(x)=\liminf _{r \rightarrow 0, y \rightarrow x} u_{h}(y),
$$

where $h \rightarrow 0$ as in the hypotheses of Theorem 1.2. By definition $\underline{u} \leq \bar{u}$ in $\bar{\Omega}$. If we show that $\bar{u}$ (resp. $\underline{u}$ ) is a generalized viscosity subsolution (resp. supersolution) of (1.3), Theorem 4.6 would imply $\bar{u} \leq \underline{u}$. Thus, $u:=\bar{u}=\underline{u}$ is a generalized viscosity solution of (1.3) and $u_{h} \rightarrow u$ uniformly in $\bar{\Omega}$. Proposition 4.7 then would imply that $u$ is a viscosity solution of (1.3).

We now sketch how to show that $\bar{u}$ is a generalized viscosity subsolution. First note that $\bar{u}$ is an upper semicontinuous function by definition, and it is also bounded since $u_{h}$ is uniformly bounded by Proposition 4.2. Take $x_{0} \in \bar{\Omega}$ and $\varphi \in C^{2}\left(B_{R}\left(x_{0}\right)\right)$ such that $\bar{u}\left(x_{0}\right)=\varphi\left(x_{0}\right)$, $\bar{u}(x)<\varphi\left(x_{0}\right)$ if $x \neq x_{0}$. We separate the proof into different cases depending of the value of the gradient of $\varphi$ at $x_{0}$ and the range of $p$.

Case 1: $\left|\nabla \varphi\left(x_{0}\right)\right| \neq 0$ or $p \geq 2$. Then, for all $x \in \bar{\Omega} \cap B_{R}\left(x_{0}\right) \backslash\left\{x_{0}\right\}$, we have that

$$
\bar{u}(x)-\varphi(x)<0=\bar{u}\left(x_{0}\right)-\varphi\left(x_{0}\right) .
$$

We claim that we can find a sequence $\left(r_{n}, y_{n}\right) \rightarrow\left(0, x_{0}\right)$ as $n \rightarrow \infty$, with $h_{n} \rightarrow 0$ as in the hypotheses of the theorem, such that

$$
u_{h_{n}}(x)-\varphi(x) \leq u_{h_{n}}\left(y_{n}\right)-\varphi\left(y_{n}\right)+e^{-1 / r_{n}} \text { for all } x \in \bar{\Omega} \cap B_{R}\left(x_{0}\right) .
$$

This can be argued for as in the proof of Theorem 2.5 ii) in [10].

Choose now $\xi_{n}:=u_{h_{n}}\left(y_{n}\right)-\varphi\left(y_{n}\right)$. We have from (4.2) that,

$$
u_{h_{n}}(x) \leq \varphi(x)+\xi_{n}+e^{-1 / r_{n}} \text { for all } x \in \bar{\Omega} \cap B_{R}\left(x_{0}\right) .
$$

Using Lemma 4.4(b)we obtain

$$
\begin{aligned}
0 & =S\left(r_{n}, h_{n}, y_{n}, u_{r_{n}}\left(y_{n}\right), u_{h_{n}}\right) \\
& =S\left(r_{n}, h_{n}, y_{n}, \varphi\left(y_{n}\right)+\xi_{n}, u_{h_{n}}\right) \\
& \geq S\left(r_{n}, h_{n}, y_{n}, \varphi\left(y_{n}\right)+\xi_{n}, \varphi+\xi_{n}+e^{-1 / r_{n}}\right) \\
& =S\left(r_{n}, h_{n}, y_{n}, \varphi\left(y_{n}\right)+\xi_{n}-e^{-1 / r_{n}}, \varphi+\xi_{n}\right) .
\end{aligned}
$$

Note that $e^{-1 / r}=o\left(r^{p}\right)$. By Lemma 4.4(b), we have

$$
\begin{aligned}
0 & \geq \liminf _{r_{n} \rightarrow 0, y_{n} \rightarrow x_{0}, \xi_{n} \rightarrow 0} S\left(r_{n}, h_{n}, y_{n}, \varphi\left(y_{n}\right)+\xi_{n}-e^{-1 / r_{n}}, \varphi+\xi_{n}\right) \\
& \geq \liminf _{r \rightarrow 0, y \rightarrow x_{0}, \xi \rightarrow 0} S\left(r, h, y, \varphi(y)+\xi-e^{-1 / r}, \varphi+\xi\right) \\
& \geq\left\{\begin{array}{cc}
-\Delta_{p} \varphi\left(x_{0}\right)-f\left(x_{0}\right) & \text { if } x_{0} \in \Omega, \\
\min \left\{-\Delta_{p} \varphi\left(x_{0}\right)-f\left(x_{0}\right), \bar{u}\left(x_{0}\right)-g\left(x_{0}\right)\right\} & \text { if } x_{0} \in \partial \Omega,
\end{array}\right.
\end{aligned}
$$


which shows that $\bar{u}$ is a viscosity subsolution and finishes the proof in this case.

Case 2: Let $p \in(1,2)$ and $\left|\nabla \varphi\left(x_{0}\right)\right|=0$ such that $\bar{u}$ is constant in some ball $B_{\rho}\left(x_{0}\right)$ for $\rho>0$ small enough. Choose $\phi(x)=\bar{u}\left(x_{0}\right)+\left|x-x_{0}\right|^{\frac{p}{p-1}+1}$. Then, we can argue as in Case 1 above that

$$
0 \geq \liminf _{r \rightarrow 0, y \rightarrow x_{0}, \xi \rightarrow 0} S\left(r, h, y, \phi(y)+\xi-e^{-1 / r}, \phi+\xi\right),
$$

which implies

$$
0 \geq \liminf _{r \rightarrow 0, y \rightarrow x_{0}} S(r, h, y, \phi(y), \phi),
$$

by the Hölder continuity of $J_{p}$. Together with Lemma A.3 this shows that

$$
-\Delta_{p} \bar{u}\left(x_{0}\right)=0 \leq f\left(x_{0}\right) .
$$

Hence, $\bar{u}$ is a classical subsolution at $x_{0}$ and thus also a viscosity subsolution.

Case 3: Let $\left|\nabla \varphi\left(x_{0}\right)\right|=0$ and assume that $\bar{u}$ is not constant in any ball $B_{\rho}\left(x_{0}\right)$. Then we may argue as in the proof of Proposition 2.4 in [3] to prove that there is a sequence $y_{k} \rightarrow 0$ such that the function $\varphi_{k}(x)=\varphi\left(x+y_{k}\right)$ touches $\bar{u}$ from above at $x_{k}=x_{0}+y_{k}$ and $\left|\nabla \varphi_{k}\left(x_{k}\right)\right| \neq 0$ for all $k$. As in Case 1 , this gives

$$
0 \geq\left\{\begin{array}{cc}
-\Delta_{p} \varphi\left(x_{k}\right)-f\left(x_{k}\right) & \text { if } x_{k} \in \Omega, \\
\min \left\{-\Delta_{p} \varphi\left(x_{k}\right)-f\left(x_{k}\right), \bar{u}\left(x_{k}\right)-g\left(x_{k}\right)\right\} & \text { if } x_{k} \in \partial \Omega,
\end{array}\right.
$$

for all $k$. Passing $k \rightarrow \infty$, we obtain

$$
\begin{aligned}
0 & \geq \limsup _{k \rightarrow \infty}\left\{\begin{array}{cc}
\left(-\Delta_{p} \varphi\left(x_{k}\right)-f\left(x_{k}\right)\right) & \text { if } x_{k} \in \Omega, \\
\min \left\{-\Delta_{p} \varphi\left(x_{k}\right)-f\left(x_{k}\right), \bar{u}\left(x_{k}\right)-g\left(x_{k}\right)\right\} & \text { if } x_{k} \in \partial \Omega,
\end{array}\right. \\
& \geq\left\{\begin{array}{cc}
\lim _{\rho \rightarrow 0} \inf _{B_{\rho}\left(x_{0}\right) \backslash\left\{x_{0}\right\}}\left(-\Delta_{p} \varphi(x)-f(x)\right) & \text { if } x_{0} \in \Omega, \\
\min \left\{\lim _{\rho \rightarrow 0} \inf _{B_{\rho}\left(x_{0}\right) \backslash\left\{x_{0}\right\}}\left(-\Delta_{p} \varphi(x)-f(x)\right), \bar{u}(x)-g(x)\right\} & \text { if } x_{0} \in \partial \Omega,
\end{array}\right.
\end{aligned}
$$

which is the desired inequality. This completes the proof.

\section{Solution of the Nonlinear System}

When we discretize the Dirichlet problem (1.3)-(1.4), we need to solve the nonlinear system (1.8)-(1.9). In contrast to the situation in [34], our system is not based on the mean value formula for the $\infty$-Laplacian which is not differentiable. Instead, it is based on an implicit and differentiable mean value property. This system is therefore well suited for Newton-Raphson, which is one of the methods we have employed. The Newton-Raphson method is fast (also mentioned by Oberman in [34]) and the number of iterations required to solve the system seems to be independent of its size, see Table 1 . However, we neither have a proof of this nor a proof of the convergence of this method. The convergence would be guaranteed for example if this is related to find the minimum of a strongly convex function. This is not the case here, since the related minimizer functional is merely convex. Nevertheless, we can give sufficient conditions for the Jacobian matrix used to be invertible. We have included this discussion in the one-dimensional setting in "Appendix 1". Since we cannot prove convergence for the Newton-Raphson method, we have also chosen to include an explicit method based on the convergence to a steady state of an evolution problem, for which we can guarantee the convergence. The convergence of this method is conditioned by the CFL-type condition 
(CFL) in Sect. 5.2. See Table 1 for a more detailed comparison between the efficiency in terms of speed of the two methods. We describe the two methods in detail below.

\subsection{Newton-Raphson}

The method we have used is the standard one. Let $F: \mathbb{R}^{k} \rightarrow \mathbb{R}^{k}$ for some $k \geq 1$. In order to solve the system

$$
F(z)=0
$$

we use the iteration

$$
z_{n+1}=z_{n}-\left(J_{F}\left(z_{n}\right)\right)^{-1} F\left(z_{n}\right) .
$$

where $J_{F}$ denotes the Jacobian matrix of the function $F$. In our particular case we have that $k=\#\left\{\tilde{G}_{h} \cap \Omega_{r}\right\}$.

Let us illustrate the form of $F$ and $J_{F}$ in the one dimensional case. Let $\gamma=\min \{\beta \in \mathbb{Z}$ : $\left.x_{\beta} \in \Omega_{r}\right\}$, and $z_{i}=U_{\gamma+i-1}$. Consider

$$
F\left(z_{1}, \ldots, z_{k}\right)=\left(\begin{array}{c}
F_{1}\left(z_{1}, \ldots, z_{k}\right) \\
F_{2}\left(z_{1}, \ldots, z_{k}\right) \\
\vdots \\
F_{k}\left(z_{1}, \ldots, z_{k}\right)
\end{array}\right)
$$

where $F_{i}: \mathbb{R}^{k} \rightarrow \mathbb{R}$ for $i=1, \ldots, k$ are given by

$$
F_{i}\left(z_{1}, \ldots, z_{k}\right)= \begin{cases}z_{i}-G_{\gamma+i-1} & \text { if } x_{\gamma+i-1} \in \partial \Omega_{r}, \\ \frac{h^{d}}{D_{d, p} \omega_{d} r^{p+d}} \sum_{x_{\alpha} \in B_{r}} J_{p}\left(z_{i+\alpha}-z_{i}\right)-f_{\gamma+i-1} & \text { if } x_{\gamma+i-1} \in \Omega .\end{cases}
$$

Let $\left(J_{F}(z)\right)_{i, j}=\left(J_{F}\left(z_{1}, \ldots, z_{k}\right)\right)_{i, j}$ denote the component of the Jacobian matrix of $F$ corresponding to the $i$-th and $j$-th column. If $i$ is such that $x_{\gamma+i-1} \in \partial \Omega_{r}$ then

$$
\left(J_{F}(z)\right)_{i, j}=\left\{\begin{array}{lll}
1 & \text { if } & j=i \\
0 & \text { if } & j \neq i
\end{array}\right.
$$

while if $x_{\gamma+i-1} \in \Omega$ then

$\left(J_{F}(z)\right)_{i, j}=\frac{(p-1) h^{d}}{D_{d, p} \omega_{d} r^{p+d}} \times \begin{cases}\left|z_{j}-z_{i}\right|^{p-2} & \text { if } j \neq i \text { and } x_{\gamma+j-1}-x_{\gamma+i-1} \in B_{r}, \\ -\sum_{x_{\alpha} \in B_{r}}\left|z_{i+\alpha}-z_{i}\right|^{p-2} & \text { if } j=i, \\ 0 & \text { otherwise. }\end{cases}$

\subsection{Explicit Method}

We consider $\left\{U^{m}\right\}_{m \in \mathbb{N}}$ to be the sequence of solutions $U^{m}: \Omega_{r}^{h} \rightarrow \mathbb{R}$ of

$$
U_{\beta}^{m+1}=U_{\beta}^{m}+\tau_{m} \Delta_{p}^{h} U_{\beta}^{m}+\tau_{m} f_{\beta}, \quad x_{\beta} \in \Omega^{h}
$$

where $U^{0}$ is some initial data, $U^{m}=G$ on $\partial \Omega_{r}^{h}$ and $\left\{\tau_{m}\right\}_{m \in \mathbb{N}}>0$ are certain discretization parameters. The idea here is that, as $m \rightarrow \infty, U^{m}$ converges to the solution $U$ of (1.8)(1.9). This convergence holds given a nonlinear counterpart to the CFL-stability condition. 
Actually, we also need to slightly modify (5.1) to ensure convergence; in words of Oberman in [33], we need to ensure that our operator is proper.

More precisely, given $\varepsilon>0$, let $\left\{\left(U_{\varepsilon}\right)^{m}\right\}_{m=1}^{\infty}$ be the solution of

$$
\left(U_{\varepsilon}\right)_{\beta}^{m+1}=\left(U_{\varepsilon}\right)_{\beta}^{m}+\tau_{m} \Delta_{p}^{h}\left(U_{\varepsilon}\right)_{\beta}^{m}-\tau_{m} \varepsilon\left(U_{\varepsilon}\right)_{\beta}^{m}+\tau f_{\beta}, \quad x_{\beta} \in \Omega^{h}
$$

subject to the same initial and boundary conditions as in (5.1). Let $U_{\varepsilon}$ be the solution of

$$
\begin{array}{lr}
-\Delta_{p}^{h}\left(U_{\varepsilon}\right)_{\beta}+\varepsilon\left(U_{\varepsilon}\right)_{\beta}=f_{\beta}, & x_{\beta} \in \Omega^{h}, \\
\left(U_{\varepsilon}\right)_{\beta}=G_{\beta}, & x_{\beta} \in \partial \Omega_{r}^{h} .
\end{array}
$$

It is standard to check, using the techniques of Sect. 4.1 that $U_{\varepsilon}$ exists, is unique and uniformly bounded in $r, h$ and $\varepsilon$. We have the following result.

Lemma 5.1 Let $p \geq 2$ and $\left\{U_{\varepsilon}^{m}\right\}_{m=1}^{\infty}$ be the solution of (5.2) with any bounded initial condition $U_{\varepsilon}^{0}$. Let also $U$ be the solution of (1.8)-(1.9). Assume that

$$
0<\tau_{m} \leq \min \left\{1, \frac{r^{p}}{(p-1) 2^{p-2} L_{m}^{p-2}} \frac{D_{d, p}}{(1+\sqrt{d})^{d}}(1-\varepsilon)\right\}
$$

with $L_{m}=\max \left(\left\|U_{\varepsilon}^{m}\right\|_{\ell^{\infty}},\left\|U_{\varepsilon}\right\|_{\ell^{\infty}}\right)$.

Then

$$
\max _{x_{\alpha} \in \Omega}\left|\left(U_{\varepsilon}\right)_{\alpha}^{m}-U_{\alpha}\right|=2 L_{0}(1-\tau \varepsilon)^{m}+o_{\varepsilon}(1),
$$

where $\tau=\inf _{m \in \mathbb{N}}\left\{\tau_{m}\right\}$.

Proof Since $U_{\varepsilon}$ is uniformly bounded in a discrete finite set, there exists a convergent subsequence $U_{\varepsilon_{j}}$ converging to some $V$ pointwise. It is also standard to show that $V$ is indeed a solution of (1.8)-(1.9). By uniqueness, $V=U$ and the full sequence $U_{\varepsilon}$ converges, i.e.,

$$
\left\|U_{\varepsilon}-U\right\|_{\infty}=o_{\varepsilon}(1) .
$$

On the other hand, by subtracting the equations for $U_{\varepsilon}$ and $\left(U_{\varepsilon}\right)^{m}$ we get

$$
\begin{aligned}
\left(U_{\varepsilon}\right)_{\beta}^{m+1}-\left(U_{\varepsilon}\right)_{\beta}= & \left(\left(U_{\varepsilon}\right)_{\beta}^{m}-\left(U_{\varepsilon}\right)_{\beta}\right)\left(1-\tau_{m} \varepsilon\right)+\tau_{m} K \\
& \times \sum_{y_{\alpha} \in B_{r}}\left(J_{p}\left(\left(U_{\varepsilon}\right)_{\beta+\alpha}^{m}-\left(U_{\varepsilon}\right)_{\beta}^{m}\right)-J_{p}\left(\left(U_{\varepsilon}\right)_{\beta+\alpha}-\left(U_{\varepsilon}\right)_{\beta}\right)\right) \\
= & \left(\left(U_{\varepsilon}\right)_{\beta}^{m}-\left(U_{\varepsilon}\right)_{\beta}\right)\left(1-\tau_{m} \varepsilon\right)+\tau_{m} K \\
& \times \sum_{y_{\alpha} \in B_{r}} J_{p}^{\prime}\left(\xi_{\alpha, \beta}\right)\left(\left(\left(U_{\varepsilon}\right)_{\beta+\alpha}^{m}-\left(U_{\varepsilon}\right)_{\beta}^{m}\right)-\left(\left(U_{\varepsilon}\right)_{\beta+\alpha}-\left(U_{\varepsilon}\right)_{\beta}\right)\right) \\
= & \left(\left(U_{\varepsilon}\right)_{\beta}^{m}-\left(U_{\varepsilon}\right)_{\beta}\right)\left(1-\tau_{m} \varepsilon-\tau_{m} K \sum_{y_{\alpha} \in B_{r}} J_{p}^{\prime}\left(\xi_{\alpha, \beta}\right)\right) \\
& +\tau_{m} K \sum_{y_{\alpha} \in B_{r}} J_{p}^{\prime}\left(\xi_{\alpha, \beta}\right)\left(\left(U_{\varepsilon}\right)_{\beta+\alpha}^{m}-\left(U_{\varepsilon}\right)_{\beta+\alpha}\right)
\end{aligned}
$$

where $K:=\frac{h^{d}}{\left|B_{r}\right| D_{d, p^{r}} r^{p}}$ and $\xi_{\alpha, \beta}$ lies between $\left(U_{\varepsilon}\right)_{\beta+\alpha}^{m}-\left(U_{\varepsilon}\right)_{\beta}^{m}$ and $\left(U_{\varepsilon}\right)_{\beta+\alpha}-\left(U_{\varepsilon}\right)_{\beta}$, so that $\left|\xi_{\alpha, \beta}\right| \leq 2 L_{m}$ and $\left|J_{p}^{\prime}\left(\xi_{\alpha, \beta}\right)\right|=(p-1)\left|\xi_{\alpha, \beta}\right|^{p-2} \leq(p-1) 2^{p-2} L_{m}^{p-2}$ since $p \geq 2$. Therefore, when $r$ is small enough 


$$
\tau_{m} K \sum_{y_{\alpha} \in B_{r}} J_{p}^{\prime}\left(\xi_{\alpha, \beta}\right) \leq(1-\varepsilon) \frac{1}{\left|B_{r}\right|} \frac{1}{(1+\sqrt{d})^{d}} \sum_{y_{\alpha} \in B_{r}} h^{d} \leq(1-\varepsilon) \frac{\left|B_{r+\sqrt{d} h}\right|}{\left|B_{r+\sqrt{d} r}\right|} \leq(1-\varepsilon),
$$

where we used (CFL) and that $h \leq r$ (since $h=o(r)$ with $r$ small enough). In this way,

$$
1-\tau_{m} \varepsilon-\tau_{m} K \sum_{y_{\alpha} \in B_{r}} J_{p}^{\prime}\left(\xi_{\alpha, \beta}\right) \geq \varepsilon\left(1-\tau_{m}\right) \geq 0 .
$$

Clearly $J_{p}^{\prime} \geq 0$, and then

$$
\begin{aligned}
\left\|\left(U_{\varepsilon}\right)^{m+1}-U_{\varepsilon}\right\|_{\ell^{\infty}} \leq & \left\|\left(U_{\varepsilon}\right)^{m}-U_{\varepsilon}\right\|_{\ell^{\infty}}\left(1-\tau_{m} \varepsilon-\tau_{m} K \sum_{y_{\alpha} \in B_{r}} J_{p}^{\prime}\left(\xi_{\alpha, \beta}\right)\right) \\
& +\tau_{m} K \sum_{y_{\alpha} \in B_{r}} J_{p}^{\prime}\left(\xi_{\alpha, \beta}\right)\left\|\left(U_{\varepsilon}\right)^{m}-U_{\varepsilon}\right\|_{\ell^{\infty}} \\
\leq & \left\|\left(U_{\varepsilon}\right)^{m}-U_{\varepsilon}\right\|_{\ell^{\infty}}\left(1-\tau_{m} \varepsilon\right) \\
\leq & \left\|\left(U_{\varepsilon}\right)^{0}-U_{\varepsilon}\right\|_{\ell^{\infty}}\left(1-\tau_{m} \varepsilon\right)^{m+1} \\
\leq & 2 L_{0}(1-\tau \varepsilon)^{m+1} .
\end{aligned}
$$

The results follows using the triangle inequality:

$$
\left\|\left(U_{\varepsilon}\right)^{m}-U\right\|_{\ell^{\infty}} \leq\left\|\left(U_{\varepsilon}\right)^{m}-U_{\varepsilon}\right\|_{\ell^{\infty}}+\left\|U_{\varepsilon}-U\right\|_{\ell^{\infty}} \leq 2 L_{0}(1-\tau \varepsilon)^{m}+o_{\varepsilon}(1) .
$$

Remark 5.2 The fact that $U_{\varepsilon}$ is uniformly bounded together with the bound $\left\|U_{\varepsilon}^{m}-U_{\varepsilon}\right\|_{\infty} \leq$ $2 L_{0}$ ensures that $L_{m}$ is uniformly bounded from above so that $\left\{\tau_{m}\right\}_{m \in N}$ can be taken uniformly bounded from below.

In the case $1<p<2$, we used a regularization of the singularity in $\Delta_{p}^{h}$ in order to make it a Lipschitz map. This could be done for example by modifying the nonlinearity with an extra approximation parameter $\delta>0$ and replacing $J_{p}$ by $J_{p}^{\delta}$ given by

$$
J_{p}^{\delta}(t)= \begin{cases}J_{p}(t+\delta)-J_{p}(\delta) & \text { if } t \geq 0 \\ J_{p}(t-\delta)-J_{p}(-\delta) & \text { if } t<0\end{cases}
$$

The drawback of this type of regularization is that the condition (CFL) becomes more and more restrictive as $\delta \rightarrow 0$. This regularization is typically used when dealing with explicit schemes for fast diffusion equations (see for example [8,9])

\subsection{Comparison Between the Solvers}

We now present a comparison of the above methods regarding the number of iterations and computational time ${ }^{2}$.

We have solved the system (1.8)-(1.9) for $p=3$, in dimension $d=1$ with $\Omega=(-1,1)$, $f \equiv 1$ and $g \equiv 0$. As starting value for the iteration we have chosen $u_{0}(x)=(1-|x|)_{+}$. Finally, for the explicit solver we have chosen $\tau$ to satisfy (CFL). We have stopped the solver when difference between two consecutive iterations is less that $10^{-16}$.

2 Naturally, this depends on the code and the computational power of the computer used, but we have chosen to include it for the sake of completeness. 
Table 1 A comparison of the efficiency of our methods used to solve the nonlinear system for $p=3$

\begin{tabular}{lllllll}
\hline$r$ & $h$ & $k$ & It-E & T-E & It-NR & T-NR \\
\hline 0.2 & 0.019037 & 127 & 4272 & 0.59 & 8 & 0.03 \\
0.1 & 0.006279 & 351 & 17475 & 9.74 & 8 & 0.1 \\
0.05 & 0.002071 & 1014 & 63164 & 166.87 & 9 & 0.84 \\
0.025 & 0.000683 & 3000 & 250901 & 3076.04 & 9 & 11.43 \\
0.0125 & 0.000025 & 8984 & $\sim 10^{7}$ & $\sim 10^{5}$ & 9 & 381.28
\end{tabular}

Here $k$ denotes the size of the system, It-E and It-NR are the number of iterations needed by the explicit and the Newton-Raphson solver respectively, and T-E and T-NR are the times (in seconds) spent to solve the system by the explicit and the Newton-Raphson solver respectively

In Table 1 we present the results for different values of $r$ and its corresponding $h$ satisfying (H) (in this case $h=\frac{r^{3 / 2+0.1}}{4}$ ).

As the table shows, the Newton-Raphson solver is fast in the sense that the number of iterations does not seem to depend on the size of the system. This is a big advantage compared to the explicit solver, for which smaller values of $r$ enforces smaller choices of $\tau$ which increase the number of iterations required substantially.

\section{Numerical Experiments}

To perform numerical experiments we need two ingredients.

(1) The explicit value of the constant $D_{d, p}$.

(2) Explicit solutions of (1.3)-(1.4) to test with.

It is standard to check that, in dimension $d=1$, we have

$$
D_{1, p}=\frac{1}{2(1+p)} \text {. }
$$

In dimension $d=2$ we have the following formula for integer numbers, that can be obtained through integration by parts. Let $p \in[2, \infty)$ and $d=2$.

(a) (Even) If $p=2 n$ for some $n \in \mathbb{N}$ then

$$
D_{2, p}=\frac{1}{2+p}\left(\prod_{i=1}^{n} \frac{2 i-1}{2 i}\right) .
$$

(b) (Odd) If $p=2 n+1$ for some $n \in \mathbb{N}$ then

$$
D_{2, p}=\frac{2}{\pi(2+p)}\left(\prod_{i=1}^{n} \frac{2 i}{2 i+1}\right) .
$$

For general dimension and $p$, one can find the following expression

$$
D_{d, p}=\frac{d}{2 \sqrt{\pi}} \cdot \frac{p-1}{d+p} \cdot \frac{\Gamma\left(\frac{d}{2}\right) \Gamma\left(\frac{p-1}{2}\right)}{\Gamma\left(\frac{d+p}{2}\right)} .
$$

As mentioned in the introduction, homogeneous problems can successfully be treated by means of the so-called normalized $p$-Laplacian, for which numerical schemes are well 
Table $2 \ell^{\infty}$-absolute error $\left\|\left(U_{\varepsilon}\right)_{h}-u\right\|_{\ell}$ and observed convergence rates $\gamma_{r}$ in $r$ and $\gamma_{h}$ in $h$ in dimension $d=1$ for problem (6.1)

\begin{tabular}{lllllllllll}
\hline & \multicolumn{3}{c}{$p=3$} & \multicolumn{3}{c}{$p=4$} \\
$r$ & $h=\frac{r^{2}}{4}$ & Error & $\gamma_{r}$ & $\gamma_{h}$ & Error & $\gamma_{r}$ & $\gamma_{h}$ & Error & $\gamma_{r}$ & $\gamma_{h}$ \\
\hline $2.00 \mathrm{e}-1$ & $1.000 \mathrm{e}-2$ & $8.46 \mathrm{e}-2$ & & & $9.13 \mathrm{e}-2$ & & & $1.23 \mathrm{e}-1$ & & \\
$1.00 \mathrm{e}-1$ & $2.500 \mathrm{e}-3$ & $4.03 \mathrm{e}-2$ & 1.07 & 0.54 & $4.35 \mathrm{e}-2$ & 1.07 & 0.54 & $5.66 \mathrm{e}-2$ & 1.12 & 0.56 \\
$5.00 \mathrm{e}-2$ & $6.250 \mathrm{e}-4$ & $2.13 \mathrm{e}-2$ & 0.92 & 0.46 & $2.27 \mathrm{e}-2$ & 0.94 & 0.47 & $2.80 \mathrm{e}-2$ & 1.02 & 0.51 \\
$2.50 \mathrm{e}-2$ & $1.563 \mathrm{e}-4$ & $1.08 \mathrm{e}-2$ & 0.97 & 0.49 & $1.17 \mathrm{e}-2$ & 0.96 & 0.48 & $2.40 \mathrm{e}-2$ & 1.00 & 0.50 \\
$1.25 \mathrm{e}-2$ & $3.906 \mathrm{e}-5$ & $5.52 \mathrm{e}-3$ & 0.97 & 0.49 & $5.86 \mathrm{e}-3$ & 1.00 & 0.50 & $6.93 \mathrm{e}-3$ & 1.02 & 0.51 \\
\hline
\end{tabular}

understood (see [32,34]). Therefore, we will focus on nonhomogeneous problems $(f \neq 0)$. We compare our numerically obtained solution with the explicit solution

$$
u(x)=\left(1-|x|^{\frac{p}{p-1}}\right) \frac{p-1}{p} \frac{1}{d^{\frac{1}{p-1}}} .
$$

Note that $u$ is a solution of

$$
\begin{cases}-\Delta_{p} u(x)=1, & x \in B_{1}, \\ u(x)=0, & x \in \partial B_{1} .\end{cases}
$$

In dimension two we will also use that for $p=4$, the smooth function $u(x, y)=x y$ solves

$$
\begin{cases}-\Delta_{p} u(x, y)=-4 x y, & (x, y) \in B_{1}, \\ u(x, y)=x y, & (x, y) \in \partial B_{1},\end{cases}
$$

and that the less regular function $u(x, y)=|x|^{\frac{4}{3}} y$ solves

$$
\begin{cases}-\Delta_{p} u(x, y)=-\frac{64}{27} y^{3}-\frac{44}{9}|x|^{2} y, & (x, y) \in B_{1}, \\ u(x, y)=|x|^{\frac{4}{3}} y, & (x, y) \in \partial B_{1} .\end{cases}
$$

\subsection{Error Analysis in Dimension $d=1$}

Here we present the results of a numerical experiment using our numerical scheme to solve problem (6.1) in dimension $d=1$ using MATLAB.

To solve the nonlinear system present in (1.8)-(1.9) we use the explicit solver given by (5.2). The parameter $\tau_{m}$ has been chosen to satisfy the (CFL), while $\varepsilon$ is chosen small enough not to interfere with the error in $h$ and $r$. We have also taken $G(x)=0$ for all $x \in \partial \Omega_{r}$ as extended boundary condition.

We have stopped the explicit solver when it has reached a numerical steady state, i.e.,

$$
\max _{x_{\alpha} \in \Omega}\left|\left(U_{\varepsilon}\right)_{\alpha}^{m+1}-\left(U_{\varepsilon}\right)_{\alpha}^{m}\right|<10^{-16} .
$$

In this case we have chosen to take $h=r^{2} / 4$ which clearly satisfy the condition $h=$ $o\left(r^{3 / 2}\right)$. The results obtained are presented in Fig. 2 and Table 2 which contain the simulations for $p=3, p=4$ and $p=10$.

It can be clearly seen that the error seems to behave linearly with $r$. This can be seen more clearly in Table 2, where we present the details of the results in Fig. 2. 

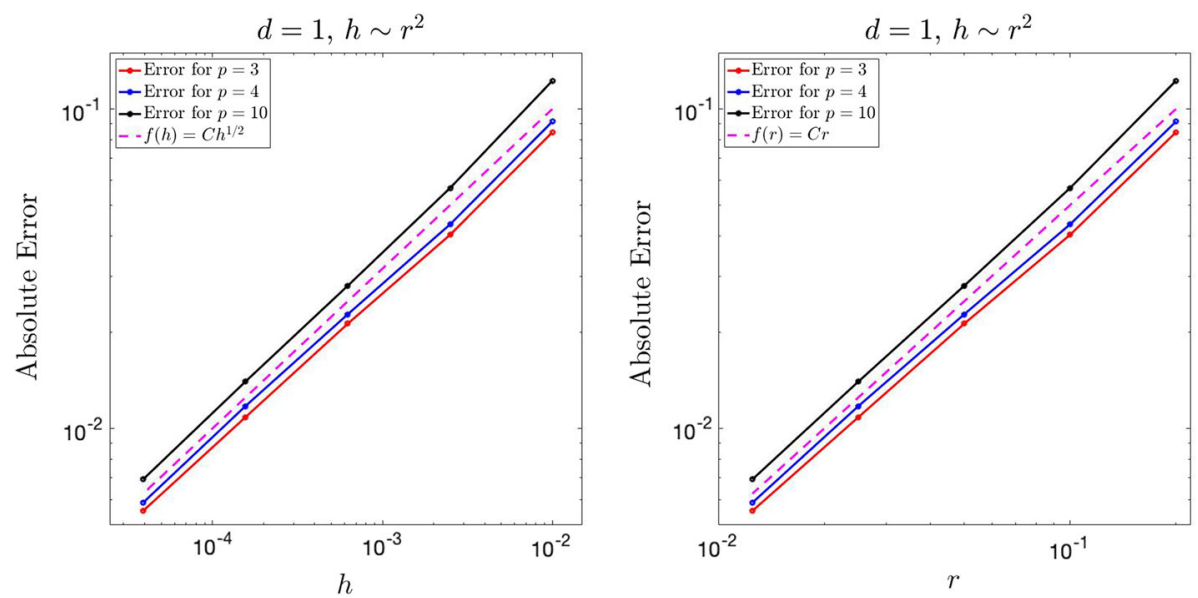

Fig. $2 \ell^{\infty}$-absolute error $\left\|\left(U_{\varepsilon}\right)_{h}-u\right\|_{\ell}$ and an approximated convergence rate in $r$ and $h$ (in rose) in dimension $d=1$ for problem (6.1)

The observed convergence rate $\gamma$ have been computed using

$$
\operatorname{error}_{j}=k\left(r_{j}\right)^{\gamma}, \quad j=0,1,2,3,4,
$$

where $r_{j}=0.2 / 2^{j}$. In this way,

$$
\gamma=\log _{2}\left(\frac{\text { error }_{j-1}}{\text { error }_{j}}\right) .
$$

\subsection{First Error Analysis in Dimension $d=2$}

We now perform numerical experiments in dimension $d=2$ for problem (6.1). We have almost the same setup as in Sect. 6.1, except that we now take $h=r^{\frac{3}{2}+0.1}$ which clearly satisfy the condition $h=o\left(r^{\frac{3}{2}}\right)$.

Again, as in the computation in dimension $d=1$, the error observed in Fig. 3 seems to decay at least linearly with $r$, despite the fact that we have taken the parameter $h$ to decay slower than before. It seems as if as long as $h=o\left(r^{3 / 2}\right)$, the choice of $h$ does not interfere with the order of convergence in $r$.

In Table 3 we observe some instabilities in the order of convergence in the simulations for big choices of $r$ and $h$. However, if we compute the order of convergence between the simulation with $r=2.00 \mathrm{e}-1$ and $r=2.50 \mathrm{e}-2$ the observed rate is

$$
\begin{aligned}
& \bar{\gamma}_{r}=\log _{8}\left(\frac{7.73 \mathrm{e}-2}{7.21 \mathrm{e}-3}\right)=1.14>1 \text { if } p=3 . \\
& \bar{\gamma}_{r}=\log _{8}\left(\frac{8.25 \mathrm{e}-2}{8.61 \mathrm{e}-3}\right)=1.09>1 \text { if } p=4 \\
& \bar{\gamma}_{r}=\log _{8}\left(\frac{1.22 \mathrm{e}-1}{1.26 \mathrm{e}-2}\right)=1.09>1 \text { if } p=10
\end{aligned}
$$

which is actually slightly better than linear in all the cases. 

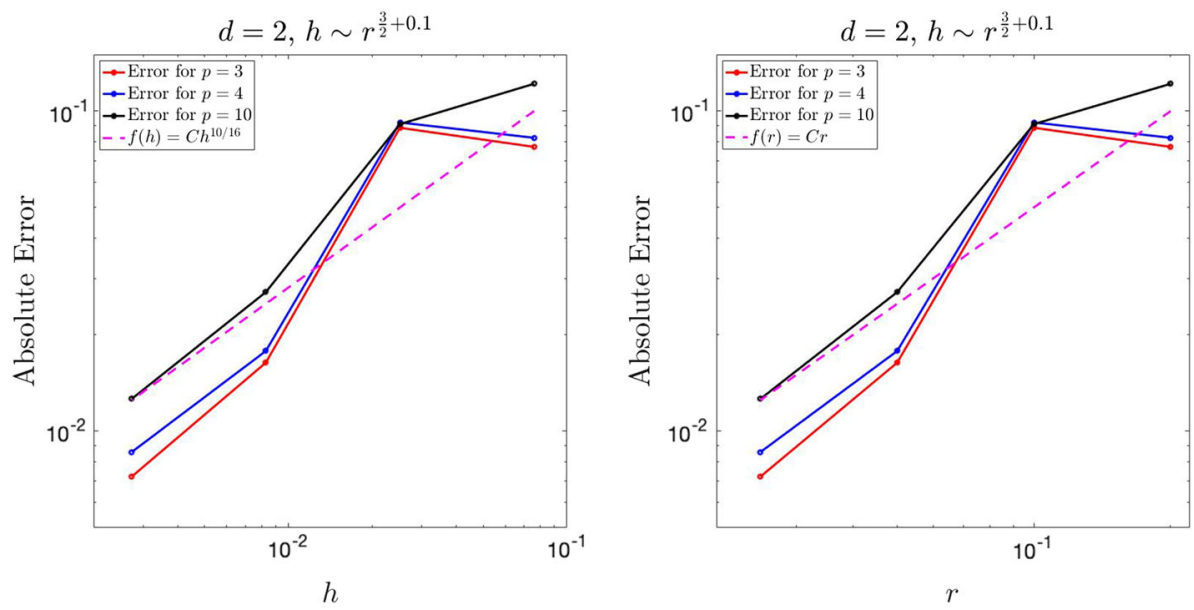

Fig. $3 \ell^{\infty}$-absolute error $\left\|\left(U_{\varepsilon}\right)_{h}-u\right\|_{\ell} \infty$ and an approximated convergence rate in $r$ and $h$ (in rose) in dimension $d=2$ for problem (6.1)

Table $3 l^{\infty}$-absolute error $\left\|\left(U_{\varepsilon}\right)_{h}-u\right\|_{\ell} \infty$ and observed convergence rates $\gamma_{r}$ in $r$ and $\gamma_{h}$ in $h$ in dimension $d=2$ for problem (6.1)

\begin{tabular}{|c|c|c|c|c|c|c|c|c|c|c|}
\hline \multirow[b]{2}{*}{$r$} & \multirow[b]{2}{*}{$h=r^{\frac{3}{2}+0.1}$} & \multicolumn{3}{|l|}{$p=3$} & \multicolumn{3}{|l|}{$p=4$} & \multicolumn{3}{|l|}{$p=10$} \\
\hline & & Error & $\gamma_{r}$ & $\gamma_{h}$ & Error & $\gamma_{r}$ & $\gamma_{h}$ & Error & $\gamma_{r}$ & $\gamma_{h}$ \\
\hline $2.00 \mathrm{e}-1$ & $7.615 \mathrm{e}-2$ & $7.73 e-2$ & & & $8.25 \mathrm{e}-2$ & & & $1.22 \mathrm{e}-1$ & & \\
\hline $1.00 \mathrm{e}-1$ & $2.512 \mathrm{e}-2$ & $8.87 \mathrm{e}-2$ & -0.20 & -0.12 & $9.21 \mathrm{e}-2$ & -0.15 & -0.9 & $9.11 \mathrm{e}-2$ & 0.41 & 0.26 \\
\hline $5.00 \mathrm{e}-2$ & $8.286 \mathrm{e}-3$ & $1.64 \mathrm{e}-2$ & 2.44 & 1.52 & $1.78 \mathrm{e}-2$ & 2.37 & 1.48 & $2.72 \mathrm{e}-2$ & 1.74 & 1.09 \\
\hline $2.50 \mathrm{e}-2$ & $2.733 \mathrm{e}-3$ & $7.21 \mathrm{e}-3$ & 1.18 & 0.74 & $8.61 \mathrm{e}-3$ & 1.05 & 0.66 & $1.26 \mathrm{e}-2$ & 1.11 & 0.69 \\
\hline
\end{tabular}

\subsection{Second Error Analysis in Dimension $d=2$}

Here, we perform numerical simulations for problems (6.2) and (6.3). We intend to illustrate that the regularity of the solution should have an impact on the order of convergence of the scheme. Note that, while the solution of (6.2) is infinitely smooth, the solution of (6.3) is no more than $C^{1,1 / 3}$. This time, we have chosen $h=r^{\frac{3}{2}+0.01}$, which satisfy the condition $h=o\left(r^{\frac{3}{2}}\right)$.

We comment now on Fig. 4. Note that for problem (6.2), where the solution is smooth, the observed error decays again linearly in $r$. On the other hand, for problem (6.3), the error decays clearly in a sublinear way. 

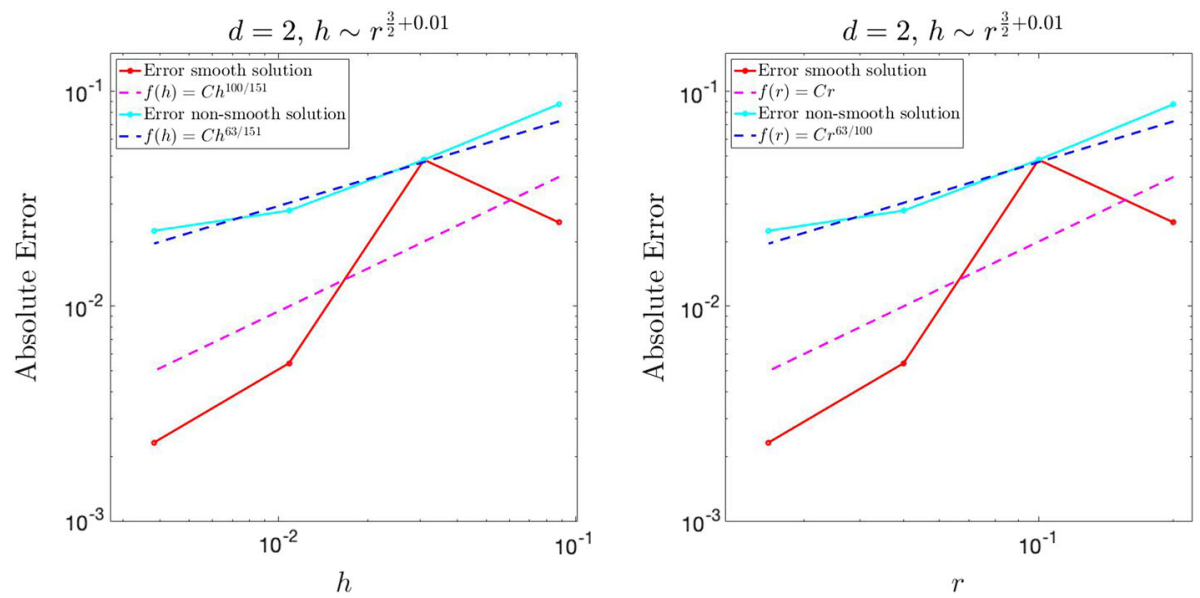

Fig. $4 \ell^{\infty}$-absolute error $\left\|\left(U_{\varepsilon}\right)_{h}-u\right\|_{\ell} \infty$ for problem (6.2) (red) and (6.3) (light blue) and respective approximated convergence rates (pink and dark blue) in dimension $d=2$

Table $4 l^{\infty}$-absolute error $\left\|\left(U_{\varepsilon}\right)_{h}-u\right\|_{\ell} \infty$ and observed convergence rates $\gamma_{r}$ in $r$ and $\gamma_{h}$ in $h$ in dimension for problem (6.2) and (6.3)

\begin{tabular}{|c|c|c|c|c|c|c|c|}
\hline \multirow[b]{2}{*}{$r$} & \multirow[b]{2}{*}{$h=r^{\frac{3}{2}+0.01}$} & \multicolumn{3}{|c|}{ Problem (6.2) } & \multicolumn{3}{|c|}{ Problem (6.3) } \\
\hline & & Error & $\gamma_{r}$ & $\gamma_{h}$ & Error & $\gamma_{r}$ & $\gamma_{h}$ \\
\hline $2.00 \mathrm{e}-1$ & $8.802 \mathrm{e}-2$ & $2.45 \mathrm{e}-2$ & & & $8.71 \mathrm{e}-2$ & & \\
\hline $1.00 \mathrm{e}-1$ & $3.090 \mathrm{e}-2$ & $4.80 \mathrm{e}-2$ & -0.97 & -0.64 & $4.80 \mathrm{e}-2$ & 0.86 & 0.57 \\
\hline $5.00 \mathrm{e}-2$ & $1.085 \mathrm{e}-2$ & $5.43 e-3$ & 3.14 & 2.08 & $2.79 \mathrm{e}-2$ & 0.79 & 0.52 \\
\hline $2.50 \mathrm{e}-2$ & $3.810 \mathrm{e}-3$ & $2.32 \mathrm{e}-3$ & 1.22 & 0.81 & $2.25 \mathrm{e}-2$ & 0.31 & 0.21 \\
\hline
\end{tabular}

In Table 4, we observe that for the smooth solution of problem (6.2), the overall convergence rates are

$$
\bar{\gamma}_{r}=\log _{8}\left(\frac{2.45 e-2}{2.32 e-3}\right)=1.13, \quad \bar{\gamma}_{h}=\frac{\bar{\gamma}_{r}}{\frac{3}{2}+0.01}=0.75,
$$

while for the non-smooth one of (6.3),

$$
\bar{\gamma}_{r}=\log _{8}\left(\frac{8.71 e-2}{2.25 e-2}\right)=0.65, \quad \bar{\gamma}_{h}=\frac{\bar{\gamma}_{r}}{\frac{3}{2}+0.01}=0.43 .
$$

Everything seems to indicate that the regularity of the solution has a significant effect in the order of convergence of the numerical scheme.

\subsection{Improvement of the Error with an Adapted Boundary Condition}

During the simulations presented in Sects. 6.1 and 6.2, we observed that the extension of $G \equiv 0$ produced a certain instability in the solution close to the boundary. Due to this fact, the maximal error is attained near the boundary. 

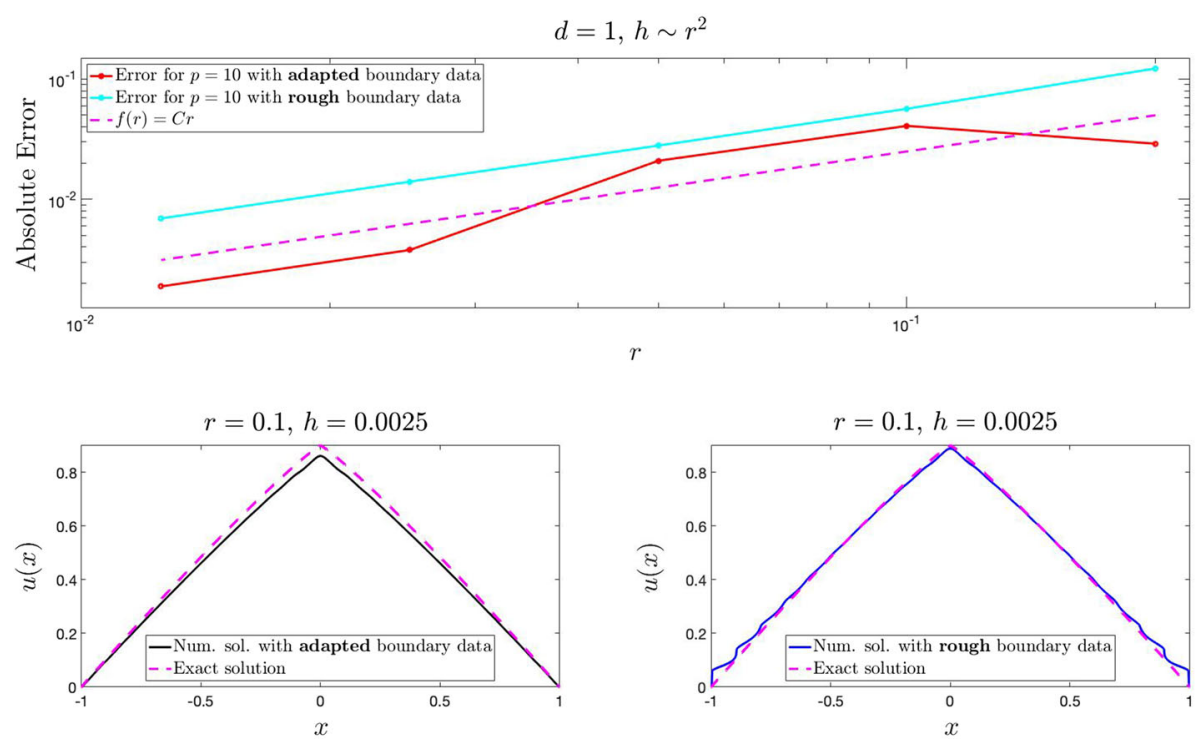

Fig. 5 Dimension $d=1$ and $p=10$. Top figure: Error analysis for the adapted and rough boundary extension $(G \equiv 0)$ and the adapted boundary extension ( $G$ given by (6.4)). Bottom figures: Representation of the numerical and real solutions for the two boundary extensions

In order to avoid this phenomenon, we have adapted the boundary condition to make the transition between the interior and the boundary smoother. We have taken

$$
G(x)=\frac{p-1}{p}\left(1-|x|^{\frac{p}{p-1}}\right) \text { for } x \in \partial \Omega_{r} .
$$

In the results presented in Fig. 5, we clearly see that the maximum error of the solution with an adapted condition comes from the middle point, which is the point where solution is the least regular, while without adaption, the error comes from the instabilities created near the boundary.

Thus, the correction seems to give a smoother transition between the interior and the extended condition. It also seems to improve the error estimate (but not the order of convergence).

The correction suggested in this section is clearly dependent on the fact that we know the explicit form of the solution. This is of course not the case in general. The behaviour near the boundary strongly depends on the boundary condition $g$ and the problem is how to choose an extended boundary condition $G$ such that the transition of the solution from $\Omega$ to $\partial \Omega_{r}$ is as smooth as possible. Unless some additional information regarding the behaviour of the solution near the boundary is available, we do not know how to do this in general.This seems to be a problem that must be handled in a problem-dependent way.

\subsection{Solution of a Fully Nonhomogeneous Problem}

Finally, we present some numerical simulations of a problem with nonhomogeneous right hand side and nonhomogeneous boundary conditions. We present the numerical solutions corresponding to problem (1.3)-(1.4) in dimension $d=2$, posed in $\Omega=B_{1}(0)$ with $f \equiv$ constant in $\Omega$ and $g(x, y)=\frac{1}{2}+x y$ on $\partial \Omega$. 

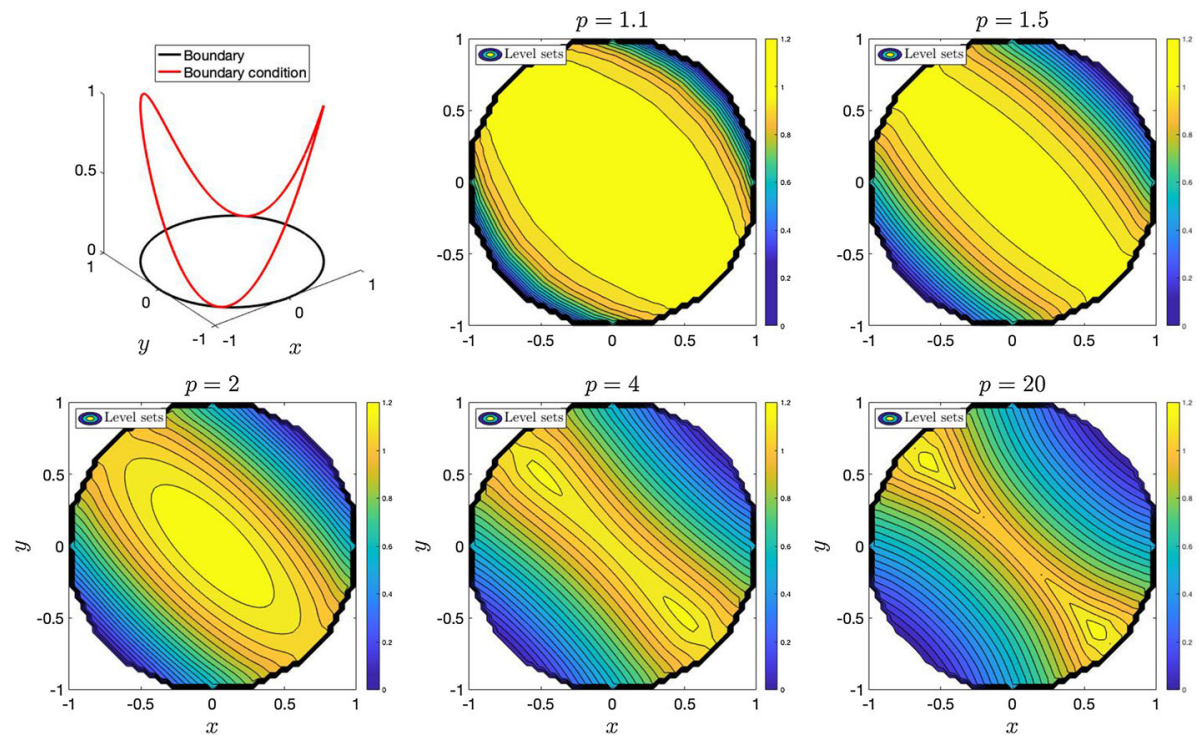

Fig. 6 Dimension $d=2$. Numerical solution of the fully nonhomogeneous problem (1.3)-(1.4) with $g(x, y)=$ $\frac{1}{2}+x y$ and $f(x, y) \equiv$ constant

The boundary condition has been extended to $\partial \Omega_{r}$ by $G(x, y)=\frac{1}{2}+x y$ and we have chosen the numerical parameters $r=0.2$ and $h=r^{2}=0.04$.

In Fig. 6, we present a level set representation of the solutions for $p=1.1, p=1.5$, $p=2, p=4$ and $p=20$ (using the regularization described at the end of Sect. 5 when $p<2$ ). Here, $h=r^{2}$ has been used, also when $p<2$ (see Remark 1.3).

Acknowledgements We would like to thank Ángel Arroyo for showing us how to explicitly compute the constants $D_{d, p}$, and to the anonymous referees for their insightful comments that certainly improved the quality of the paper.

\section{Author Contributions Not applicable.}

Funding Open Access funding provided thanks to the CRUE-CSIC agreement with Springer Nature. F. del Teso was partially supported by PGC2018-094522-B-I00 from the MICINN of the Spanish Government. E. Lindgren is supported by the Swedish Research Council, grant no. 2017-03736.

\section{Declarations}

Conflict of interest Not applicable.

Availability of data and material Not applicable.

Code availability Custom code in Matlability Available in this link.

Ethics approval Not applicable.

Consent to participate Not applicable.

Consent for publication Not applicable. 
Open Access This article is licensed under a Creative Commons Attribution 4.0 International License, which permits use, sharing, adaptation, distribution and reproduction in any medium or format, as long as you give appropriate credit to the original author(s) and the source, provide a link to the Creative Commons licence, and indicate if changes were made. The images or other third party material in this article are included in the article's Creative Commons licence, unless indicated otherwise in a credit line to the material. If material is not included in the article's Creative Commons licence and your intended use is not permitted by statutory regulation or exceeds the permitted use, you will need to obtain permission directly from the copyright holder. To view a copy of this licence, visit http://creativecommons.org/licenses/by/4.0/.

\section{Appendix 1: Pointwise Inequalities and a Special Limit}

Lemma A.1 Let $p \geq 2$. Then

$$
|| a+\left.b\right|^{p-2}(a+b)-|a|^{p-2} a\left|\leq C \max (|a|,|a+b|)^{p-2}\right| b \mid,
$$

where $C=C(p)$.

Proof It follows from the fact that

$$
|a+b|^{p-2}(a+b)-|a|^{p-2} a=(p-1) \int_{0}^{b}|a+s|^{p-2} \mathrm{~d} s .
$$

The following inequality is Lemma 3.4 in [18].

Lemma A.2 Let $p \in(1,2)$. Then

$$
|| a+\left.b\right|^{p-2}(a+b)-|a|^{p-2} a\left|\leq C(|a|+|b|)^{p-2}\right| b \mid .
$$

Here $C=C(p)$.

We also need the following lemma in the proof of convergence.

Lemma A.3 Assume $p \in(1,2)$, (H) and let $\phi(x)=|x|^{\beta}$ with $\beta>p /(p-1)$. Then

$$
\lim _{r \rightarrow 0, x \rightarrow 0} \Delta_{p}^{h} \phi(x)=0 .
$$

Proof If $x=0$, we have $\left|\phi\left(x+y_{\alpha}\right)-\phi(x)\right|=|y|^{\beta}=o\left(\left|y_{\alpha}\right|^{\frac{p}{p-1}}\right)$. Then

$$
\left|\Delta_{p}^{h} \phi(x)\right| \leq J_{p}\left(o\left(|r|^{\frac{p}{p-1}}\right)\right) \frac{1}{\left|B_{r}\right| r^{p}} \sum_{y_{\alpha} \in B_{r}} h^{d}=o_{r}(1) .
$$

Assume now that $x \neq 0$ so that $\nabla \phi(x) \neq 0$. We can use the symmetry of $J_{p}\left(y_{\alpha} \cdot \nabla \phi(x)\right)$ for $y_{\alpha} \in B_{r}$ and Lemma A.2 to conclude that

$$
\begin{gathered}
\left|\sum_{y_{\alpha} \in B_{r}} J_{p}\left(\phi\left(x+y_{\alpha}\right)-\phi(x)\right) \mathrm{d} y\right| \\
\leq\left|\sum_{y_{\alpha} \in B_{r}} J_{p}\left(y_{\alpha} \cdot \nabla \phi(x)+\frac{1}{2} y_{\alpha}^{T} D^{2} \phi\left(\xi_{\alpha}\right) y_{\alpha}\right)\right| \\
\leq\left.\left.\sum_{y_{\alpha} \in B_{r}}|| y_{\alpha} \cdot \nabla \phi(x)|+| y_{\alpha}\right|^{2} \sup _{\xi \in B_{r}(x)}\left|D^{2} \phi(\xi)\right|\right|^{p-2} \\
\left|y_{\alpha}\right|^{2} \sup _{\xi \in B_{r}(x)}\left|D^{2} \phi(\xi)\right| \mathrm{d} y .
\end{gathered}
$$


We may assume that $x$ lies in the $e_{1}$-direction and write $\nabla \phi(x)=\beta|x|^{\beta-1} e_{1}:=c e_{1}$ for some $c>0$. We now claim that ${ }^{3}$

$$
\begin{aligned}
\Delta_{p}^{h} \phi(x) & \lesssim \frac{c^{p-2} h^{d}}{\left|B_{r}\right|} \sum_{y_{\alpha} \in B_{r}}|| \hat{y}_{\alpha} \cdot e_{1}\left|+c^{-1} r \sup _{\xi \in B_{r}(x)}\right| D^{2} \phi(\xi)||^{p-2} \sup _{\xi \in B_{r}(x)}\left|D^{2} \phi(\xi)\right| \\
& \leq \frac{c^{p-2} h^{d}}{\left|B_{r}\right|} \sum_{y_{\alpha} \in B_{r}}|| \hat{y}_{\alpha} \cdot e_{1}\left|+c^{-1} r C(|x|+r)^{\beta-2}\right|^{p-2} C(|x|+r)^{\beta-2} \\
& \leq \frac{c^{p-2}}{\left|B_{r}\right|}\left(\int_{B_{2 r}}|| \hat{y} \cdot e_{1}\left|+c^{-1} r C(|x|+r)^{\beta-2}\right|^{p-2} C(|x|+r)^{\beta-2} \mathrm{~d} y\right)+o_{r+|x|}(1) .
\end{aligned}
$$

Once this is proved, it only remains to to prove that the first term in (A.1) goes to zero. This is the estimate obtained on page 24 in the proof of Lemma A.4 in [10], with the small difference that we here integrate over $B_{2 r}$ instead of $B_{r}$.

We now explain how to obtain (A.1). Fix $r$ and $x$ and consider the function

$$
f(y)=|| \hat{y} \cdot e_{1}\left|+c^{-1} r C(|x|+r)^{\beta-2}\right|^{p-2} C(|x|+r)^{\beta-2} .
$$

The midpoint quadrature rule applied to $f(y)$ yields

$$
\left|\int_{\tilde{B}_{r}} f(\hat{y}) d y-h^{d} \sum_{y_{\alpha} \in B_{r}} f\left(\hat{y}_{\alpha}\right)\right| \lesssim\left\|D^{2} f\right\|_{L^{\infty}\left(\tilde{B}_{r}\right)} h^{2}\left|\tilde{B}_{r}\right| .
$$

Upon multiplying with $\frac{c^{p-2}}{\left|B_{r}\right|}$, inserting $f(y)$ and rearranging, we obtain

$$
\begin{aligned}
& \frac{c^{p-2} h^{d}}{\left|B_{r}\right|} \sum_{y_{\alpha} \in B_{r}}|| \hat{y}_{\alpha} \cdot e_{1}\left|+c^{-1} r C(|x|+r)^{\beta-2}\right|^{p-2} C(|x|+r)^{\beta-2} \\
& \lesssim \frac{c^{p-2}}{\left|B_{r}\right|} \int_{\tilde{B}_{r}}|| \hat{y} \cdot e_{1}\left|+c^{-1} r C(|x|+r)^{\beta-2}\right|^{p-2} C(|x|+r)^{\beta-2} \mathrm{~d} y+c^{p-2}\left\|D^{2} f\right\|_{L^{\infty}\left(\tilde{B}_{r}\right)} h^{2} \\
& \lesssim \frac{c^{p-2}}{\left|B_{r}\right|} \int_{B_{2 r}}|| \hat{y} \cdot e_{1}\left|+c^{-1} r C(|x|+r)^{\beta-2}\right|^{p-2} C(|x|+r)^{\beta-2} \mathrm{~d} y+c^{p-2}\left\|D^{2} f\right\|_{L^{\infty}\left(\tilde{B}_{r}\right)} h^{2}
\end{aligned}
$$

where we use that $\tilde{B}_{r} \subset B_{r+\sqrt{d} h} \subset B_{2 r}$ for $r$ small enough, since $h=o(r)$. The only thing left is to prove that the last term is $o_{r+|x|}(1)$. Differentiation of $f$ yields

$$
\left\|D^{2} f\right\|_{L^{\infty}\left(\tilde{B}_{r}\right)} \lesssim\left|c^{-1} r C(|x|+r)^{\beta-2}\right|^{p-4} C(|x|+r)^{\beta-2} .
$$

Since $h=o\left(r^{p /(p-1)}\right)=o\left(r^{3 / 2}\right), \beta-2>0, p-3<0$ and $c=\beta|x|^{\beta-1}$, we obtain

$$
\begin{aligned}
c^{p-2}\left\|D^{2} f\right\|_{L^{\infty}\left(\tilde{B}_{r}\right)} h^{2} & \lesssim c^{p-2} c^{4-p}(|x|+r)^{(\beta-2)(p-3)} r^{p-1} \\
& =c^{2}(|x|+r)^{(\beta-2)(p-3)} r^{p-1} \\
& =|x|^{2(\beta-1)}(|x|+r)^{(\beta-2)(p-3)} r^{p-1}
\end{aligned}
$$

If $|x| \leq r$ then

$|x|^{2(\beta-1)}(|x|+r)^{(\beta-2)(p-3)} r^{p-1} \lesssim r^{2(\beta-1)} r^{(\beta-2)(p-3)} r^{p-1}=r^{(\beta-1)(2+p-3)-p+3+p-1}=r^{3}$.

3 Here $a \lesssim b$ stands for $a \leq C b$ where $C$ is a constant that may depend on $p$ and $d$ but not on $r, h$ or $x$. 
Likewise, if $r \leq|x|$ then

$$
|x|^{2(\beta-1)}(|x|+r)^{(\beta-2)(p-3)} r^{p-1} \lesssim|x|^{2(\beta-1)}|x|^{(\beta-2)(p-3)}|x|^{p-1}=|x|^{3} .
$$

This shows (A.1) and concludes the proof.

\section{Appendix 2: Invertibility of the Jacobian}

In this part we discuss the invertibility of the Jacobian used in the Newton-Raphson method formulated in Sect. 5. We have the following general result.

Lemma B.1 Let $A=\left(A_{i j}\right)$ be an $n \times n$ matrix satisfying

(a) $\left|A_{i i}\right|>0$ for all $i=1, \ldots, n$;

(b) $\sum_{j=1, j \neq i}^{n}\left|A_{i j}\right| \leq\left|A_{i i}\right|$ for all $i=1, \ldots, n$;

(c) $\sum_{j=2}^{n}\left|A_{1 j}\right|<\left|A_{11}\right|$

(d) For all $i=2, \ldots, n$ and for some $j<i$ we have either

$$
\sum_{j=1, j \neq i}^{n}\left|A_{i j}\right|<\left|A_{i i}\right|
$$

or

$$
A_{i j} \neq 0
$$

Then A is invertible.

Proof We argue towards a contradiction. Assume there is a non-zero $x=\left(x_{1}, \ldots, x_{n}\right) \in \mathbb{R}^{n}$ such that $A x=0$. We may also assume that $\|x\|_{\infty}=1$.

We observe that if $\left|x_{i}\right|=1$ for $i=1$ or $i$ such that property (B.1) holds, then

$$
\left|A_{i i}\right|=\left|A_{i i} x_{i}\right|=\left|\sum_{j=1, j \neq i}^{n} A_{i j} x_{j}\right| \leq \sum_{j=1, j \neq i}^{n}\left|A_{i j} x_{j}\right|<\left|A_{i i}\right|,
$$

which is a contradiction. In the last inequality, we used property (c) or (B.1).

Assume now that $\left|x_{i}\right|<1$ for $i=1, \ldots, k-1$ and that $\left|x_{k}\right|=1$. If $k$ is such that (B.1) holds, then arguing as in (B.3), we arrive at a contradiction. Therefore, $k$ must be such that (B.2) holds, which means that there is $\ell<k$ such that $A_{k \ell} \neq 0$. Then

$$
\begin{aligned}
\left|A_{k k}\right|=\left|A_{k k} x_{k}\right| & =\left|A_{k \ell} x_{\ell}+\sum_{j=1, j \neq k, \ell}^{n} A_{k j} x_{j}\right| \\
& \leq\left|A_{k \ell}\right|\left|x_{\ell}\right|+\sum_{j=1, j \neq k, \ell}^{n}\left|A_{k j}\right|\left|x_{j}\right| \\
& <A_{k \ell}+\sum_{j=1, j \neq k, \ell}^{n}\left|A_{k j}\right| \\
& \leq\left|A_{k k}\right|,
\end{aligned}
$$


where we have used that $\left|x_{\ell}\right|<1$ and that $\left|x_{j}\right| \leq 1$ in the third inequality, and property 2) in the last inequality. This is a contradiction.

We now review the form of the Jacobian in the Newton-Raphson method (see Sect. 5) and give sufficient conditions for it to satisfies the assumptions of Lemma B.1. Recall that the Jacobian is given by the matrix $A_{i j}$ as follows. If $i$ is such that $x_{\gamma+i-1} \in \partial \Omega_{r}$ then

$$
A_{i j}= \begin{cases}1 & \text { if } j=i \\ 0 & \text { if } j \neq i\end{cases}
$$

while if $x_{\gamma+i-1} \in \Omega$ then

$$
A_{i j}=\frac{(p-1) h^{d}}{D_{d, p} \omega_{d} r^{p+d}} \times \begin{cases}\left|z_{j}-z_{i}\right|^{p-2} & \text { if } j \neq i \text { and } x_{\gamma+j-1}-x_{\gamma+i-1} \in B_{r}, \\ -\sum_{x_{\alpha} \in B_{r}}\left|z_{i+\alpha}-z_{i}\right|^{p-2} & \text { if } j=i \\ 0 & \text { otherwise. }\end{cases}
$$

For simplicity, let

$$
h(t)=\frac{(p-1) h^{d}}{D_{d, p} \omega_{d} r^{p+d}}|t|^{p-2} .
$$

Assume that $B_{r}$ contains $2 L+1$ elements. Then the first $L$ rows are simply given by

$$
(1,0, \ldots, 0), \quad(0,1,0, \ldots, 0), \cdots, \underbrace{0, \ldots, 0}_{L \text { zeros }}, 1,0, \ldots) .
$$

Row number $L+1+m$ for $m=0, \ldots, k-2 L-1$ is (transposed)

$$
\left(\begin{array}{c}
\vdots \\
0 \\
h\left(z_{2+m}-z_{L+1+m}\right) \\
\vdots \\
h\left(z_{L+m}-z_{L+1+m}\right) \\
-\sum_{j=2}^{2 L} h\left(z_{j+m}-z_{L+1+m}\right) \\
h\left(z_{L+2+m}-z_{L+1+m}\right) \\
\vdots \\
h\left(z_{2 L+m}-z_{L+1+m}\right) \\
0 \\
\vdots
\end{array}\right)
$$

and this continues until the last $L$ rows, that are of the form

$$
(0, \ldots, 0,1, \underbrace{0, \ldots, 0}_{L \text { zeros }}), \cdots \quad, \quad(0, \ldots, 1,0), \quad(0, \ldots, 0,1) .
$$

We will now give conditions on the values $z_{1}, \ldots, z_{k}$, assuring that the assumptions of Lemma B.1 are satisfied. 
Lemma B.2 Assume that for each $z_{i}$, with $i=L+1, \ldots, k-L$, there is $j$ between $i-L+1$ and $i-1$ such that $z_{j} \neq z_{i}$. Then the matrix A defined by (B.4)-(B.5) is invertible.

Proof Let us go through the assumptions of Lemma B.1. The diagonal elements consists of $1 \mathrm{~s}$ or are of the form

$$
-\sum_{j=2+m}^{2 L+m} h\left(z_{L+1+m}-z_{j}\right) .
$$

By assumption, at least one of the terms of the sum is non-zero. It is therefore clear that assumption (a) is satisfied. Assumptions (b) and (c) are trivially satisfied. Regarding assumption (d), it is clear that (B.2) is always satisfied.

We note that the condition given in Lemma B.2 is saying that the solution is not constant in any $B_{r}$ neighbourhood to the left of any point. This is a very reasonable assumption, since, for example, for non-vanishing right hand side $f$, this is true for smooth solutions. Also for $f=0$ and $g \neq 0$, in dimension two, this is the case.

\section{References}

1. Amghibech, S.: Eigenvalues of the discrete $p$-Laplacian for graphs. Ars Combin. 67, 283-302 (2003)

2. Arroyo, A., Llorente, J.G.: On the asymptotic mean value property for planar $p$-harmonic functions. Proc. Amer. Math. Soc. 144(9), 3859-3868 (2016)

3. Attouchi, A., Ruosteenoja, E.: Remarks on regularity for $p$-Laplacian type equations in non-divergence form. J. Differ. Equ. 265(5), 1922-1961 (2018)

4. Barles, G., Souganidis, P.E.: Convergence of approximation schemes for fully nonlinear second order equations. Asymptotic Anal. 4(3), 271-283 (1991)

5. Barrett, J.W., Liu, W.B.: Finite element approximation of the $p$-Laplacian. Math. Comp. 61(204), 523-537 (1993)

6. Bucur,C., Squassina,M.: An asymptotic expansion for the fractional $p$-laplacian and gradient dependent nonlocal operators. Commun. Contemp. Math. (online ready), 2021

7. Codenotti, L., Lewicka, M., Manfredi, J.: Discrete approximations to the double-obstacle problem and optimal stopping of tug-of-war games. Trans. Amer. Math. Soc. 369(10), 7387-7403 (2017)

8. del Teso, F., Endal, J., Jakobsen, E.R.: Robust numerical methods for nonlocal (and local) equations of porous medium type. Part II: Schemes and experiments. SIAM J. Numer. Anal. 56(6), 3611-3647 (2018)

9. del Teso, F., Endal, J., Jakobsen, E.R.: Robust numerical methods for nonlocal (and local) equations of porous medium type. Part I: Theory. SIAM J. Numer. Anal. 57(5), 2266-2299 (2019)

10. del Teso,F., Lindgren,E.: A mean value formula for the variational $p$-Laplacian. NoDEA Nonlinear Differ. Equ. Appl. 28(3), 2021

11. del Teso,F., Manfredi,J.J., Parviainen,M.: Convergence of dynamic programming principles for the $p$ laplacian. Adv. Calc. Var. (online ready), 2021

12. Elmoataz, A., Toutain, M., Tenbrinck, D.: On the $p$-laplacian and $\infty$-laplacian on graphs with applications in image and data processing. SIAM J. Imaging Sci. 8(4), 2412-2451 (2015)

13. Ferreira, R., dePablo, A., Pérez-Llanos, M.: Numerical blow-up for the $p$-Laplacian equation with a source. Comput. Methods Appl. Math. 5(2), 137-154 (2005)

14. Glowinski,R., Marrocco,A.: Sur l'approximation, par éléments finis d'ordre un, et la résolution, par pénalisation-dualité, d'une classe de problèmes de Dirichlet non linéaires. Rev. Française Automat. Informat. Recherche Opérationnelle Sér. Rouge Anal. Numér., 9(R-2):41-76, 1975

15. Julin, V., Juutinen, P.: A new proof for the equivalence of weak and viscosity solutions for the $p$-Laplace equation. Commun. Partial Differ. Equ. 37(5), 934-946 (2012)

16. Kawohl, B., Manfredi, J., Parviainen, M.: Solutions of nonlinear PDEs in the sense of averages. J. Math. Pures Appl. 97(2), 173-188 (2012)

17. Kim, K.Y.: Error estimates for a mixed finite volume method for the $p$-Laplacian problem. Numer. Math. 101(1), 121-142 (2005)

18. Korvenpää, J., Kuusi, T., Lindgren, E.: Equivalence of solutions to fractional $p$-Laplace type equations. J. Math. Pures Appl. 9(132), 1-26 (2019) 
19. Lewicka,M.: Random tug of war games for the $p$-laplacian: $1<p<+\infty$. Preprint: arXiv:1810.03413v, 2018

20. Lewicka, M., Manfredi, J.J.: Game theoretical methods in PDEs. Boll. Unione Mat. Ital. 7(3), 211-216 (2014)

21. Lewicka, M., Manfredi, J.J.: The obstacle problem for the $p$-laplacian via optimal stopping of tug-of-war games. Probab. Theory Related Fields 167(1-2), 349-378 (2017)

22. Lindqvist,P.: Notes on the Stationary $p$-Laplace Equation. Springer Briefs in Mathematics. Springer, Cham (2019)

23. Lindqvist, P., Manfredi, J.: On the mean value property for the $p$-Laplace equation in the plane. Proc. Amer. Math. Soc. 144(1), 143-149 (2016)

24. Liu, W., Yan, N.: Quasi-norm a priori and a posteriori error estimates for the nonconforming approximation of p-Laplacian. Numer. Math. 89(2), 341-378 (2001)

25. Liu, W., Yan, N.: On quasi-norm interpolation error estimation and a posteriori error estimates for $p$ Laplacian. SIAM J. Numer. Anal. 40(5), 1870-1895 (2002)

26. Liu, W.B., Barrett, J.W.: A remark on the regularity of the solutions of the $p$-Laplacian and its application to their finite element approximation. J. Math. Anal. Appl. 178(2), 470-487 (1993)

27. Loisel, S.: Efficient algorithms for solving the $p$-Laplacian in polynomial time. Numer. Math. 146(2), 369-400 (2020)

28. Manfredi, J.J., Oberman, A.M., Sviridov, A.P.: Nonlinear elliptic partial differential equations and $p$ harmonic functions on graphs. Differ. Integral Equ. 28(1-2), 79-102 (2015)

29. Manfredi, J.J., Parviainen, M., Rossi, J.D.: An asymptotic mean value characterization for $p$-harmonic functions. Proc. Amer. Math. Soc. 138(3), 881-889 (2010)

30. Manfredi, J.J., Parviainen, M., Rossi, J.D.: Dynamic programming principle for tug-of-war games with noise. ESAIM Control Optim. Calc. Var. 18(1), 81-90 (2012)

31. Manfredi,J.J., Parviainen,M., Rossi,J.D.: On the definition and properties of $p$-harmonious functions. Ann. Sc. Norm. Super. Pisa Cl. Sci. (5), 11(2):215-241, 2012

32. Oberman, A.M.: A convergent difference scheme for the infinity Laplacian: construction of absolutely minimizing Lipschitz extensions. Math. Comp. 74(251), 1217-1230 (2005)

33. Oberman, A.M.: Convergent difference schemes for degenerate elliptic and parabolic equations: Hamilton-Jacobi equations and free boundary problems. SIAM J. Numer. Anal. 44(2), 879-895 (2006)

34. Oberman, A.M.: Finite difference methods for the infinity Laplace and $p$-Laplace equations. J. Comput. Appl. Math. 254, 65-80 (2013)

35. Peres, Y., Schramm, O., Sheffield, S., Wilson, D.B.: Tug-of-war and the infinity Laplacian. J. Amer. Math. Soc. 22(1), 167-210 (2009)

36. Wang, Y.-Z., Huang, H.: Eigenvalue estimates of the p-laplacian on finite graphs. Differ. Geometry Appl. 74, 101697 (2021)

37. Yamasaki, M.: Discrete potentials on an infinite network. Mem. Fac. Lit. Sci. Shimane Univ. 13, 31-44 (1979)

Publisher's Note Springer Nature remains neutral with regard to jurisdictional claims in published maps and institutional affiliations. 